\title{
OPTIMIZED SCHWARZ METHODS FOR MAXWELL'S EQUATIONS*
}

\author{
V. DOLEAN ${ }^{\dagger}$, M.J. GANDER ${ }^{\ddagger}$, AND L. GERARDO-GIORDA $^{\S}$
}

\begin{abstract}
Over the last two decades, classical Schwarz methods have been extended to systems of hyperbolic partial differential equations, using characteristic transmission conditions, and it has been observed that the classical Schwarz method can be convergent even without overlap in certain cases. This is in strong contrast to the behavior of classical Schwarz methods applied to elliptic problems, for which overlap is essential for convergence. More recently, optimized Schwarz methods have been developed for elliptic partial differential equations. These methods use more effective transmission conditions between subdomains than the classical Dirichlet conditions, and optimized Schwarz methods can be used both with and without overlap for elliptic problems. We show here why the classical Schwarz method applied to both the time harmonic and time discretized Maxwell's equations converges without overlap: the method has the same convergence factor as a simple optimized Schwarz method for a scalar elliptic equation. Based on this insight, we develop an entire new hierarchy of optimized overlapping and nonoverlapping Schwarz methods for Maxwell's equations with greatly enhanced performance compared to the classical Schwarz method. We also derive for each algorithm asymptotic formulas for the optimized transmission conditions, which can easily be used in implementations of the algorithms for problems with variable coefficients. We illustrate our findings with numerical experiments.
\end{abstract}

Key words. Schwarz algorithms, optimized transmission conditions, Maxwell's equations

AMS subject classifications. $65 \mathrm{M} 55,65 \mathrm{~F} 10,65 \mathrm{~N} 22$

DOI. $10.1137 / 080728536$

1. Introduction. Schwarz algorithms experienced a second youth over the last decades when distributed computers became more and more powerful and available. Fundamental convergence results for the classical Schwarz methods were derived for many partial differential equations and can now be found in several authoritative reviews [3, 41, 42] and books [34, 33, 39]. The Schwarz methods were also extended to systems of partial differential equations, such as the time harmonic Maxwell's equations $[12,8]$, the time discretized Maxwell's equations [38], or to linear elasticity $[18,19]$, but much less is known about the behavior of the Schwarz methods applied to hyperbolic systems of equations. This is true, in particular, for the Euler equations, to which the Schwarz algorithm was first applied in [31, 32], where classical (characteristic) transmission conditions are used at the interfaces, or with more general transmission conditions in [7]. The analysis of such algorithms applied to systems proved to be very different from the scalar case; see [14, 15].

Over the last decade, a new class of overlapping Schwarz methods was developed for scalar partial differential equations, namely, the optimized Schwarz methods. These methods are based on a classical overlapping domain decomposition, but they use more effective transmission conditions than the classical Dirichlet conditions at the interfaces between subdomains. New transmission conditions were originally proposed for three different reasons: first, to obtain Schwarz algorithms that are convergent without overlap; see [28] for Robin conditions. The second motivation for

\footnotetext{
* Received by the editors June 25, 2008; accepted for publication (in revised form) December 5, 2008; published electronically May 7, 2009.

http://www.siam.org/journals/sisc/31-3/72853.html

$\dagger$ Laboratoire J.-A. Dieudonné, Univ. de Nice Sophia-Antipolis, Nice, France (dolean@unice.fr).

¥Section de Mathématiques, Université de Genève, CP 64, 1211 Genève, Switzerland (martin. gander@unige.ch).

$\S^{\S}$ Department of Mathematics, University of Trento, Trento, Italy (gerardo@science.unitn.it).
} 
changing the transmission conditions was to obtain a convergent Schwarz method for the Helmholtz equation, where the classical overlapping Schwarz algorithm is not convergent. As a remedy, approximate radiation conditions were introduced in $[10,12]$. The third motivation was that the convergence rate of the classical Schwarz method is rather slow and too strongly dependent on the size of the overlap. In a short note on nonlinear problems [26], Hagstrom, Tewarson, and Jazcilevich introduced Robin transmission conditions between subdomains and suggested nonlocal operators for the best performance. In [4], these optimal, nonlocal transmission conditions were developed for advection-diffusion problems, with local approximations for small viscosity, and low order frequency approximations were proposed in $[29,9]$. In [35], one can find low-frequency approximations of absorbing boundary conditions for the Euler equations. Independently, at the algebraic level, generalized coupling conditions were introduced in $[37,36]$ for discrete overlapping Schwarz methods. Optimized transmission conditions for the best performance of the Schwarz algorithm in a given class of local transmission conditions were first introduced for advection-diffusion problems in [27], for the Helmholtz equation in [6, 24], and for Laplace's equation in [17]. For complete results and attainable performance for a symmetric, positive definite problem, see [20], and for time dependent problems, see $[23,21]$. The purpose of this paper is to design and analyze a family of optimized overlapping and nonoverlapping Schwarz methods for Maxwell's equations, both for the case of time discretized and time harmonic problems, and to provide explicit formulas for the optimized parameters in the transmission conditions of each algorithm in the family. These formulas can then easily be used in implementations for Maxwell's equations with variable coefficients. As we will see, one member of this family reduces in the case of no overlap and constant coefficients to an algorithm in a curl-curl formulation of Maxwell's equations, proposed in [1] based on [5], which already greatly enhanced the performance compared to the classical approaches in $[12,8]$.

This paper is organized as follows: in section 2, we present Maxwell's equations and a reformulation thereof with characteristic variables used in our analysis. In section 3, we treat the case of time harmonic solutions. We show that the classical Schwarz method for Maxwell's equations, which uses characteristic Dirichlet transmission conditions between subdomains, is convergent even without overlap. Exploiting a relation with an optimized Schwarz method applied to a Helmholtz equation allows us to develop an entirely new hierarchy of optimized Schwarz methods for Maxwell's equations with greatly enhanced performance, both with and without overlap. A similar relation has been used in [13] for the Cauchy-Riemann equations. In section 4, we present and analyze the corresponding hierarchy of optimized Schwarz methods for time discretizations of Maxwell's equations. We then show in section 5 numerical experiments in two and three spatial dimensions, both for the time harmonic and time discretized cases, which illustrate the performance of the new optimized Schwarz methods for Maxwell's equations. We also include as an application the cooking of a chicken in a microwave oven, a problem with variable coefficients. In section 6 , we summarize our findings and conclude with an outlook on future research directions.

2. Maxwell's equations. The hyperbolic system of Maxwell's equations describes the propagation of electromagnetic waves. It is given by

$$
-\varepsilon \frac{\partial \mathcal{E}}{\partial t}+\operatorname{curl} \mathcal{H}-\sigma \mathcal{E}=\mathcal{J}, \quad \mu \frac{\partial \mathcal{H}}{\partial t}+\operatorname{curl} \mathcal{E}=0,
$$


where $\mathcal{E}=\left(\mathcal{E}_{1}, \mathcal{E}_{2}, \mathcal{E}_{3}\right)^{T}$ and $\mathcal{H}=\left(\mathcal{H}_{1}, \mathcal{H}_{2}, \mathcal{H}_{3}\right)^{T}$ denote the electric and magnetic fields, respectively, $\varepsilon$ is the electric permittivity, $\mu$ is the magnetic permeability, $\sigma$ is the electric conductivity, and $\mathcal{J}$ is the applied current density. We assume the applied current density to be divergence free, that is, $\operatorname{div} \mathcal{J}=0$. Denoting the vector of physical unknowns by

$$
\boldsymbol{u}=\left(\mathcal{E}_{1}, \mathcal{E}_{2}, \mathcal{E}_{3}, \mathcal{H}_{1}, \mathcal{H}_{2}, \mathcal{H}_{3}\right)^{T},
$$

Maxwell's equations (2.1) can be rewritten in the form

$$
\left(G+G_{0} \partial_{t}\right) \boldsymbol{u}+G_{x} \partial_{x} \boldsymbol{u}+G_{y} \partial_{y} \boldsymbol{u}+G_{z} \partial_{z} \boldsymbol{u}=(\mathcal{J} ; \mathbf{0}),
$$

where the coefficient matrices are

$$
G=\left[\begin{array}{ll}
\sigma I_{3} & \\
& 0_{3}
\end{array}\right], G_{0}=\left[\begin{array}{ll}
\varepsilon I_{3} & \\
& \mu I_{3}
\end{array}\right], G_{l}=\left[\begin{array}{cc} 
& N_{l} \\
-N_{l} &
\end{array}\right], \quad l=x, y, z,
$$

where $0_{3}$ (resp., $I_{3}$ ) represent the $3 \times 3$ zero (resp., identity) matrix, and the matrices $N_{l}, l=x, y, z$, are given by

$$
N_{x}=\left[\begin{array}{ccc}
0 & 0 & 0 \\
0 & 0 & 1 \\
0 & -1 & 0
\end{array}\right], \quad N_{y}=\left[\begin{array}{ccc}
0 & 0 & -1 \\
0 & 0 & 0 \\
1 & 0 & 0
\end{array}\right], \quad N_{z}=\left[\begin{array}{ccc}
0 & 1 & 0 \\
-1 & 0 & 0 \\
0 & 0 & 0
\end{array}\right]
$$

For any unit vector $\boldsymbol{n}=\left(n_{1}, n_{2}, n_{3}\right),\|\boldsymbol{n}\|=1$, we can define the characteristic matrix of system (2.3) by

$$
C(\boldsymbol{n})=G_{0}^{-1}\left(n_{1}\left[\begin{array}{ll} 
& N_{x} \\
-N_{x} &
\end{array}\right]+n_{2}\left[\begin{array}{ll} 
& N_{y} \\
-N_{y} &
\end{array}\right]+n_{3}\left[\begin{array}{ll}
-N_{z} & N_{z}
\end{array}\right]\right),
$$

whose eigenvalues are the characteristic speed of propagation along the direction $\boldsymbol{n}$. A direct calculation shows that the matrix $C(\boldsymbol{n})$ has real eigenvalues

$$
\lambda_{1,2}=-c, \quad \lambda_{3,4}=0, \quad \lambda_{5,6}=c,
$$

with $c=\frac{1}{\sqrt{\varepsilon \mu}}$ being the wave speed. This implies that Maxwell's equations are hyperbolic, since the eigenvalues are real, but not strictly hyperbolic, since the eigenvalues are not distinct; see [2]. For the special case of the normal vector $\boldsymbol{n}=(1,0,0)$, which we will use extensively later, we obtain

$$
C(\boldsymbol{n})=\left(\begin{array}{cc} 
& \frac{1}{\varepsilon} N_{x} \\
-\frac{1}{\mu} N_{x} &
\end{array}\right)
$$

whose matrix of eigenvectors is given by

$$
L=\left[\begin{array}{cccccc}
0 & 0 & 0 & 1 & 0 & 0 \\
-Z & 0 & 0 & 0 & Z & 0 \\
0 & Z & 0 & 0 & 0 & -Z \\
0 & 0 & 1 & 0 & 0 & 0 \\
0 & 1 & 0 & 0 & 0 & 1 \\
1 & 0 & 0 & 0 & 1 & 0
\end{array}\right]
$$

Copyright $\odot$ by SIAM. Unauthorized reproduction of this article is prohibited. 
where $Z=\sqrt{\frac{\mu}{\varepsilon}}$ denotes the impedance. This leads to the characteristic variables $\boldsymbol{w}=\left(w_{1}, w_{2}, w_{3}, w_{4}, w_{5}, w_{6}\right)^{T}=L^{-1} \boldsymbol{u}$ associated with the direction $\boldsymbol{n}$, where

$$
\begin{aligned}
& w_{1}=-\frac{1}{2}\left(\frac{1}{Z} \mathcal{E}_{2}-\mathcal{H}_{3}\right), \quad w_{2}=\frac{1}{2}\left(\frac{1}{Z} \mathcal{E}_{3}+\mathcal{H}_{2}\right), \quad w_{3}=\mathcal{H}_{1}, \\
& w_{4}=\mathcal{E}_{1}, \quad w_{5}=\frac{1}{2}\left(\frac{1}{Z} \mathcal{E}_{2}+\mathcal{H}_{3}\right), \quad w_{6}=-\frac{1}{2}\left(\frac{1}{Z} \mathcal{E}_{3}-\mathcal{H}_{2}\right) .
\end{aligned}
$$

In the following, we will denote by $\boldsymbol{w}_{+}, \boldsymbol{w}_{0}$, and $\boldsymbol{w}_{-}$the characteristic variables associated with the negative, zero, and positive eigenvalues, respectively, that is,

$$
\boldsymbol{w}_{-}=\left(w_{1}, w_{2}\right)^{T}, \quad \boldsymbol{w}_{0}=\left(w_{3}, w_{4}\right)^{T}, \quad \boldsymbol{w}_{+}=\left(w_{5}, w_{6}\right)^{T} .
$$

Imposing classical or characteristic boundary conditions on a boundary with unit outward normal vector $\boldsymbol{n}=(1,0,0)$ means to impose Dirichlet conditions on the incoming characteristic variables $\boldsymbol{w}_{-}$. For a general normal vector $\boldsymbol{n}$, this is equivalent to imposing the impedance condition (see [2])

$$
\mathcal{B}_{\boldsymbol{n}}(\mathcal{E}, \mathcal{H}):=\boldsymbol{n} \times \frac{\mathcal{E}}{Z}+\boldsymbol{n} \times(\mathcal{H} \times \boldsymbol{n})=\boldsymbol{s} .
$$

3. Time harmonic solutions. Time harmonic solutions of Maxwell's equations are complex-valued static vector fields $\boldsymbol{E}$ and $\boldsymbol{H}$ such that the dynamic fields

$$
\mathcal{E}(\boldsymbol{x}, t)=\mathcal{R} e(\boldsymbol{E}(\boldsymbol{x}) \exp (i \omega t)), \quad \mathcal{H}(\boldsymbol{x}, t)=\mathcal{R} e(\boldsymbol{H}(\boldsymbol{x}) \exp (i \omega t))
$$

satisfy Maxwell's equations (2.1). The positive real parameter $\omega$ is called the pulsation of the harmonic wave. The harmonic solutions $\boldsymbol{E}$ and $\boldsymbol{H}$ satisfy the time harmonic Maxwell's equations

$$
-i \omega \varepsilon \boldsymbol{E}+\operatorname{curl} \boldsymbol{H}-\sigma \boldsymbol{E}=\boldsymbol{J}, \quad i \omega \mu \boldsymbol{H}+\operatorname{curl} \boldsymbol{E}=\mathbf{0} .
$$

3.1. Classical and optimized Schwarz algorithm. We consider now the problem (3.1) in a bounded domain $\Omega$, with either Dirichlet conditions on the tangent electric field or impedance conditions, on $\partial \Omega$, in order to obtain a well-posed problem; see [30]. In order to explain the classical Schwarz algorithm for Maxwell's equation, we decompose the domain into two overlapping subdomains $\Omega_{1}$ and $\Omega_{2}$ as illustrated in Figure 3.1. The generalization of the algorithm formulation to the case of many subdomains does not present any difficulties. The classical Schwarz algorithm then

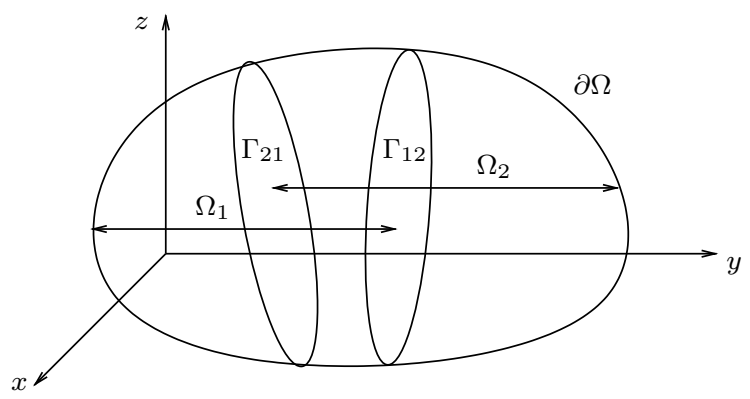

FIG. 3.1. Overlapping domain decomposition. 
solves for $n=1,2, \ldots$ the subdomain problems

$$
\begin{aligned}
-i \omega \varepsilon \boldsymbol{E}^{1, n}+\operatorname{curl} \boldsymbol{H}^{1, n}-\sigma \boldsymbol{E}^{1, n} & =\boldsymbol{J} & & \text { in } \Omega_{1}, \\
i \omega \mu \boldsymbol{H}^{1, n}+\operatorname{curl} \boldsymbol{E}^{1, n} & =\mathbf{0} & & \text { in } \Omega_{1}, \\
\mathcal{B}_{\boldsymbol{n}_{1}}\left(\boldsymbol{E}^{1, n}, \boldsymbol{H}^{1, n}\right) & =\mathcal{B}_{\boldsymbol{n}_{1}}\left(\boldsymbol{E}^{2, n-1}, \boldsymbol{H}^{2, n-1}\right) & & \text { on } \Gamma_{12}, \\
-i \omega \varepsilon \boldsymbol{E}^{2, n}+\operatorname{curl} \boldsymbol{H}^{2, n}-\sigma \boldsymbol{E}^{2, n} & =\boldsymbol{J} & & \text { in } \Omega_{2}, \\
i \omega \mu \boldsymbol{H}^{2, n}+\operatorname{curl} \boldsymbol{E}^{2, n} & =\mathbf{0} & & \text { in } \Omega_{2}, \\
\mathcal{B}_{\boldsymbol{n}_{2}}\left(\boldsymbol{E}^{2, n}, \boldsymbol{H}^{2, n}\right) & =\mathcal{B}_{\boldsymbol{n}_{2}}\left(\boldsymbol{E}^{1, n-1}, \boldsymbol{H}^{1, n-1}\right) & & \text { on } \Gamma_{21},
\end{aligned}
$$

where $\Gamma_{12}=\partial \Omega_{1} \cap \Omega_{2}, \Gamma_{21}=\partial \Omega_{2} \cap \Omega_{1}$, and $\mathcal{B}_{\boldsymbol{n}_{j}}, j=1,2$, denotes the impedance boundary conditions defined in (2.6). On the physical part of the boundary, the given boundary conditions are imposed. While the choice of transmission conditions $\mathcal{B}_{\boldsymbol{n}_{j}}$ is natural in the view of the hyperbolic nature of the problem, we will see in our analysis that there are better choices for the performance of the algorithm, based on the notion of absorbing boundary conditions. This leads to the so-called optimized Schwarz methods

$$
\begin{aligned}
-i \omega \varepsilon \boldsymbol{E}^{1, n}+\operatorname{curl} \boldsymbol{H}^{1, n}-\sigma \boldsymbol{E}^{1, n} & =\boldsymbol{J} & & \text { in } \Omega_{1}, \\
i \omega \mu \boldsymbol{H}^{1, n}+\operatorname{curl} \boldsymbol{E}^{1, n} & =\mathbf{0} & & \text { in } \Omega_{1}, \\
\left(\mathcal{B}_{\boldsymbol{n}_{1}}+\mathcal{S}_{1} \mathcal{B}_{\boldsymbol{n}_{2}}\right)\left(\boldsymbol{E}^{1, n}, \boldsymbol{H}^{1, n}\right) & =\left(\mathcal{B}_{\boldsymbol{n}_{1}}+\mathcal{S}_{1} \mathcal{B}_{\boldsymbol{n}_{2}}\right)\left(\boldsymbol{E}^{2, n-1}, \boldsymbol{H}^{2, n-1}\right) & & \text { on } \Gamma_{12}, \\
-i \omega \varepsilon \boldsymbol{E}^{2, n}+\operatorname{curl} \boldsymbol{H}^{2, n}-\sigma \boldsymbol{E}^{2, n} & =\boldsymbol{J} & & \text { in } \Omega_{2}, \\
i \omega \mu \boldsymbol{H}^{2, n}+\operatorname{curl} \boldsymbol{E}^{2, n} & =\mathbf{0} & & \text { in } \Omega_{2}, \\
\left(\mathcal{B}_{\boldsymbol{n}_{2}}+\mathcal{S}_{2} \mathcal{B}_{\boldsymbol{n}_{1}}\right)\left(\boldsymbol{E}^{2, n}, \boldsymbol{H}^{2, n}\right) & =\left(\mathcal{B}_{\boldsymbol{n}_{2}}+\mathcal{S}_{2} \mathcal{B}_{\boldsymbol{n}_{1}}\right)\left(\boldsymbol{E}^{1, n-1}, \boldsymbol{H}^{1, n-1}\right) & & \text { on } \Gamma_{21},
\end{aligned}
$$

where $\mathcal{S}_{j}, j=1,2$, are tangential, possibly pseudodifferential operators we will study in what follows in order to obtain various optimized Schwarz methods.

3.2. Convergence analysis for the classical Schwarz algorithm. We now study properties of the classical Schwarz algorithm (3.2). We use Fourier analysis and, thus, assume that the coefficients are constant, and the domain on which the original problem is posed is $\Omega=\mathbb{R}^{3}$, in which case we need for Maxwell's equations the Silver-Müller radiation condition

$$
\lim _{r \rightarrow \infty} r(\boldsymbol{H} \times \boldsymbol{n}-\boldsymbol{E})=0,
$$

where $r=|\boldsymbol{x}|$ and $\boldsymbol{n}=\boldsymbol{x} /|\boldsymbol{x}|$, in order to obtain well-posed problems; see [30]. The two subdomains are now half-spaces

$$
\Omega_{1}=(0, \infty) \times \mathbb{R}^{2}, \quad \Omega_{2}=(-\infty, L) \times \mathbb{R}^{2},
$$

the interfaces are $\Gamma_{12}=\{L\} \times \mathbb{R}^{2}$ and $\Gamma_{21}=\{0\} \times \mathbb{R}^{2}$, and the overlap is $L \geq 0$. We denote by $k_{y}$ and $k_{z}$ the Fourier variables corresponding to a transform with respect to $y$ and $z$, respectively, and $|\boldsymbol{k}|^{2}=k_{y}^{2}+k_{z}^{2}$.

Theorem 3.1. For any given initial guess $\left(\boldsymbol{E}^{1,0} ; \boldsymbol{H}^{1,0}\right) \in\left(L^{2}\left(\Omega_{1}\right)\right)^{6},\left(\boldsymbol{E}^{2,0} ; \boldsymbol{H}^{2,0}\right) \in$ $\left(L^{2}\left(\Omega_{2}\right)\right)^{6}$, the classical Schwarz algorithm (3.2) with overlap $L \geq 0$, including the nonoverlapping case, is for $\sigma>0$ convergent in $\left(L^{2}\left(\Omega_{1}\right)\right)^{6} \times\left(L^{2}\left(\Omega_{2}\right)\right)^{6}$, and the convergence factor for each Fourier mode $\boldsymbol{k}$ is

$$
\rho_{c l a}(\boldsymbol{k}, \tilde{\omega}, \sigma, Z, L)=\left|\frac{\sqrt{|\boldsymbol{k}|^{2}-\tilde{\omega}^{2}+i \tilde{\omega} \sigma Z}-i \tilde{\omega}}{\sqrt{|\boldsymbol{k}|^{2}-\tilde{\omega}^{2}+i \tilde{\omega} \sigma Z}+i \tilde{\omega}} e^{-\sqrt{|\boldsymbol{k}|^{2}-\tilde{\omega}^{2}+i \tilde{\omega} \sigma Z} L}\right|,
$$

where $\tilde{\omega}=\omega \sqrt{\varepsilon \mu}$ and $Z=\sqrt{\frac{\mu}{\varepsilon}}$ is the impedance as before. 
Proof. Because of linearity, it suffices to analyze the convergence to the zero solution when the right-hand side vanishes. Performing a Fourier transform of system (3.1) in the $y$ and $z$ directions, the first and the fourth equations provide an algebraic expression for $\hat{E}_{1}$ and $\hat{H}_{1}$, which is in agreement with the fact that these are the characteristic variables associated with the null eigenvalue. Inserting these expressions into the remaining Fourier transformed equations, we obtain the first order system

$$
\partial_{x}\left(\begin{array}{l}
\hat{E}_{2} \\
\hat{E}_{3} \\
\hat{H}_{2} \\
\hat{H}_{3}
\end{array}\right)+\left[\begin{array}{cccc}
0 & 0 & -\frac{k_{y} k_{z}}{i \omega \varepsilon+\sigma} & \frac{-\tilde{\omega}^{2}+k_{y}^{2}+i \omega \mu \sigma}{i \omega \varepsilon+\sigma} \\
0 & 0 & \frac{\tilde{\omega}^{2}-k_{z}^{2}-i \omega \mu \sigma}{i \omega \varepsilon+\sigma} & \frac{k_{y} k_{z}}{i \omega \varepsilon+\sigma} \\
\frac{k_{y} k_{z}}{i \omega \mu} & \frac{\tilde{\omega}^{2}-k_{y}^{2}-i \omega \mu \sigma}{i \omega \mu} & 0 & 0 \\
\frac{-\tilde{\omega}^{2}+k_{z}^{2}+i \omega \mu \sigma}{i \omega \mu} & -\frac{k_{y} k_{z}}{i \omega \mu} & 0 & 0
\end{array}\right]\left(\begin{array}{l}
\hat{E}_{2} \\
\hat{E}_{3} \\
\hat{H}_{2} \\
\hat{H}_{3}
\end{array}\right)=\left(\begin{array}{l}
0 \\
0 \\
0 \\
0
\end{array}\right) .
$$

The eigenvalues of the matrix in (3.7) and their corresponding eigenvectors are (3.8)

$$
\lambda_{1,2}^{T H}=-\sqrt{|\boldsymbol{k}|^{2}-\tilde{\omega}^{2}+i \omega \mu \sigma}, \quad \boldsymbol{v}_{1}=\left(\begin{array}{c}
\frac{k_{y} k_{z}}{(i \omega \varepsilon+\sigma) \lambda} \\
\frac{-\tilde{\omega}^{2}+k_{z}^{2}+i \omega \mu \sigma}{(i \omega \varepsilon+\sigma) \lambda} \\
1 \\
0
\end{array}\right), \quad \boldsymbol{v}_{2}=\left(\begin{array}{c}
\frac{\tilde{\omega}^{2}-k_{y}^{2}-i \omega \mu \sigma}{(i \omega \varepsilon+\sigma) \lambda} \\
-\frac{k_{y} k_{z}}{(i \omega \varepsilon+\sigma) \lambda} \\
0 \\
1
\end{array}\right)
$$

and

$$
\lambda_{3,4}^{T H}=\sqrt{|\boldsymbol{k}|^{2}-\tilde{\omega}^{2}+i \omega \mu \sigma}, \quad \boldsymbol{v}_{3}=\left(\begin{array}{c}
-\frac{k_{y} k_{z}}{(i \omega \varepsilon+\sigma) \lambda} \\
\frac{\tilde{\omega}^{2}-k_{z}^{2}-i \omega \mu \sigma}{(i \omega \varepsilon+\sigma) \lambda} \\
1 \\
0
\end{array}\right), \quad \boldsymbol{v}_{4}=\left(\begin{array}{c}
\frac{k_{y}^{2}-\tilde{\omega}^{2}+i \omega \mu \sigma}{(i \omega \varepsilon+\sigma) \lambda} \\
\frac{k_{y} k_{z}}{(i \omega \varepsilon+\sigma) \lambda} \\
0 \\
1
\end{array}\right),
$$

where we set $\lambda:=\sqrt{|\boldsymbol{k}|^{2}-\tilde{\omega}^{2}+i \omega \mu \sigma}$. Because of the radiation condition, the solutions of system (3.7) in $\Omega_{l}, l=1,2$, are given by

$$
\left(\hat{E}_{2}^{1} ; \hat{E}_{3}^{1} ; \hat{H}_{2}^{1} ; \hat{H}_{3}^{1}\right)=\left(\alpha_{1} \boldsymbol{v}_{1}+\alpha_{2} \boldsymbol{v}_{2}\right) e^{\lambda(x-L)}, \quad\left(\hat{E}_{2}^{2} ; \hat{E}_{3}^{2} ; \hat{H}_{2}^{2} ; \hat{H}_{3}^{2}\right)=\left(\beta_{1} \boldsymbol{v}_{3}+\beta_{2} \boldsymbol{v}_{4}\right) e^{-\lambda x},
$$

where the coefficients $\alpha_{j}$ and $\beta_{j}(j=1,2)$ are uniquely determined by the transmission conditions. At the $n$th step of the Schwarz algorithm, the coefficients $\boldsymbol{\alpha}=\left(\alpha_{1}, \alpha_{2}\right)$ and $\boldsymbol{\beta}=\left(\beta_{1}, \beta_{2}\right)$ satisfy the system

$$
\boldsymbol{\alpha}^{n}=A_{1}^{-1} A_{2} e^{-\lambda L} \boldsymbol{\beta}^{n-1}, \quad \boldsymbol{\beta}^{n}=B_{1}^{-1} B_{2} e^{-\lambda L} \boldsymbol{\alpha}^{n-1},
$$

where the matrices in the iteration are given by

$$
\begin{aligned}
& A_{1}=\left[\begin{array}{cc}
-k_{y} k_{z} & k_{y}^{2}-\tilde{\omega}^{2}+i \tilde{\omega} \lambda+\sigma Z(\lambda+i \tilde{\omega}) \\
k_{z}^{2}-\tilde{\omega}^{2}+i \tilde{\omega} \lambda+\sigma Z(\lambda+i \tilde{\omega}) & -k_{y} k_{z}
\end{array}\right], \\
& k_{y} k_{z} \\
& A_{2}=\left[\begin{array}{cc}
2 & -k_{y}^{2}+\tilde{\omega}^{2}+i \tilde{\omega} \lambda+\sigma Z(\lambda-i \tilde{\omega}) \\
-k_{z}^{2}+\tilde{\omega}^{2}+i \tilde{\omega} \lambda+\sigma Z(\lambda-i \tilde{\omega}) & k_{y} k_{z}
\end{array}\right]
\end{aligned}
$$

and where $B_{l}=A_{l}, l=1,2$. A complete iteration over two steps of the Schwarz algorithm leads then to

$$
\boldsymbol{\alpha}^{n+1}=\left(A_{1}^{-1} A_{2}\right)^{2} e^{-2 \lambda L} \boldsymbol{\alpha}^{n-1}, \quad \boldsymbol{\beta}^{n+1}=\left(A_{1}^{-1} A_{2}\right)^{2} e^{-2 \lambda L} \boldsymbol{\beta}^{n-1},
$$


and we obtain the iteration matrix

$$
R=\left(A_{1}^{-1} A_{2}\right)^{2} e^{-2 \lambda L}=\left[\begin{array}{cc}
\frac{|\boldsymbol{k}|^{4}+2 \lambda \sigma Z\left(k_{y}^{2}-k_{z}^{2}\right)+\lambda^{2} \sigma^{2} Z^{2}}{(\lambda+i \tilde{\omega})^{2}(\lambda+i \tilde{\omega}+\sigma Z)^{2}} & \frac{4 k_{y} k_{z} \lambda \sigma Z}{(\lambda+i \tilde{\omega})^{2}(\lambda+i \tilde{\omega}+\sigma Z)^{2}} \\
\frac{4 k_{y} k_{z} \lambda \sigma Z}{(\lambda+i \tilde{\omega})^{2}(\lambda+i \tilde{\omega}+\sigma Z)^{2}} & \frac{|\boldsymbol{k}|^{4}+2 \lambda \sigma Z\left(k_{z}^{2}-k_{y}^{2}\right)+\lambda^{2} \sigma^{2} Z^{2}}{(\lambda+i \tilde{\omega})^{2}(\lambda+i \tilde{\omega}+\sigma Z)^{2}}
\end{array}\right] e^{-2 \lambda L} .
$$

Now, by the definition of $\lambda$, we have $|\boldsymbol{k}|^{2}=\lambda^{2}+\tilde{\omega}^{2}-i \tilde{\omega} \sigma Z$, and, thus, this matrix can be rewritten in factored form:

$$
R=\left(\frac{\lambda-i \tilde{\omega}}{\lambda+i \tilde{\omega}}\right)^{2} e^{-2 \lambda L} I d+\frac{4 \lambda \sigma Z}{(\lambda+i \tilde{\omega})^{2}(\lambda+i \tilde{\omega}+\sigma Z)^{2}}\left[\begin{array}{cc}
-k_{z}^{2} & k_{y} k_{z} \\
k_{y} k_{z} & -k_{y}^{2}
\end{array}\right] e^{-2 \lambda L} .
$$

The convergence factor $\rho_{\text {cla }}$ of the algorithm is given by the square root of the spectral radius of the matrix $R$, whose eigenvalues are $\left(\frac{\lambda-i \tilde{\omega}}{\lambda+i \tilde{\omega}}\right)^{2} e^{-2 \lambda L}$ and $\left(\frac{\lambda-i \tilde{\omega}-\sigma Z}{\lambda+i \tilde{\omega}+\sigma Z}\right)^{2} e^{-2 \lambda L}$. Since $\sigma \geq 0$, a direct computation shows that the convergence factor is given by the first eigenvalue, which leads to (3.6), and when $\sigma \neq 0$, a straightforward computation shows that $\rho_{\text {cla }}(\boldsymbol{k})<1$ for all Fourier modes $\boldsymbol{k}$.

If $\sigma=0$, the convergence factor becomes

$$
\rho_{\text {cla }}(\boldsymbol{k}, \tilde{\omega}, 0, Z, L)= \begin{cases}\left|\frac{\sqrt{\tilde{\omega}^{2}-|\boldsymbol{k}|^{2}}-\tilde{\omega}}{\sqrt{\tilde{\omega}^{2}-|\boldsymbol{k}|^{2}}+\tilde{\omega}}\right| & \text { for }|\boldsymbol{k}|^{2} \leq \tilde{\omega}^{2} \\ e^{-\sqrt{|\boldsymbol{k}|^{2}-\tilde{\omega}^{2}} L} & \text { for }|\boldsymbol{k}|^{2}>\tilde{\omega}^{2}\end{cases}
$$

In this case, we obtain for $|\boldsymbol{k}|^{2}=\tilde{\omega}^{2}$ that the convergence factor equals 1 , independent of the overlap, which indicates that the algorithm has convergence problems for $\sigma=0$ when used in the iterative form described here. Convergence can still be proved in the case of a bounded domain with suitable boundary conditions; see [12]. In addition, in practice, Schwarz methods are often used as preconditioners for Krylov methods, which can handle isolated problems in the spectrum. We also see from the convergence factor (3.13) that in the case $\sigma=0$ the overlap is necessary for the convergence of the evanescent modes, $|\boldsymbol{k}|^{2}>\tilde{\omega}^{2}$. Without overlap, $L=0$, we have $\rho_{\text {cla }}(\boldsymbol{k})<1$ only for the propagative modes, $|\boldsymbol{k}|^{2}<\tilde{\omega}^{2}$, and $\rho_{\text {cla }}(\boldsymbol{k})=1$ when $|\boldsymbol{k}|^{2} \geq \tilde{\omega}^{2}$.

Very similar observations were made in the analysis of optimized Schwarz methods for the Helmholtz equation in [24]. If one applies to the Helmholtz equation

$$
\left(\Delta+\tilde{\omega}^{2}\right) u=f \quad \text { in } \Omega=\mathbb{R}^{3},
$$

with Sommerfeld radiation conditions $\lim _{r \rightarrow \infty} r\left(\frac{\partial u}{\partial r}-i \tilde{\omega} u\right)=0$ and the same two subdomain decomposition (3.5), the somewhat particular overlapping Schwarz method (note the unequal treatment in the transmission conditions)

$$
\begin{aligned}
& \left(\tilde{\omega}^{2}+\Delta\right) u_{1}^{1, n}=f \quad \text { in } \Omega_{1}, \quad\left(\tilde{\omega}^{2}+\Delta\right) u_{1}^{2, n}=f \quad \text { in } \Omega_{2}, \\
& u_{1}^{1, n}=u_{1}^{2, n-1} \text { on } \Gamma_{12}, \quad\left(\partial_{x}-i \tilde{\omega}\right) u_{1}^{2, n}=\left(\partial_{x}-i \tilde{\omega}\right) u_{1}^{1, n-1} \text { on } \Gamma_{21},
\end{aligned}
$$

then one obtains precisely the same convergence factor (3.13). The classical overlapping Schwarz algorithm with characteristic transmission conditions (3.2) for Maxwell's equations is, thus, equivalent to the particular overlapping Schwarz method (3.15) for the Helmholtz problem when $\sigma=0$. This particular Schwarz method is a very simple variant of an optimized Schwarz method, where one has replaced only one of 
the Dirichlet transmission conditions with a better one adapted for low frequencies. There are much better transmission conditions for Helmholtz problems as shown in [24]. These conditions are based on approximations of transparent boundary conditions, which we will study in the next subsection for Maxwell's equations.

3.3. Transparent boundary conditions. To design optimized Schwarz methods for Maxwell's equations, we derive now transparent boundary conditions for those equations, following the approach in [25]. We consider the time harmonic Maxwell's equations (3.1) on the domains $\Omega_{1}=(-\infty, L) \times \mathbb{R}^{2}$ and $\Omega_{2}=(0, \infty) \times \mathbb{R}^{2}$ with righthand sides $\boldsymbol{J}_{1,2}$ compactly supported in $\Omega_{1,2}$, together with the boundary conditions

$$
\left(\boldsymbol{w}_{+}^{2}+\mathcal{S}_{1} \boldsymbol{w}_{-}^{2}\right)(0, y, z)=0, \quad\left(\boldsymbol{w}_{-}^{1}+\mathcal{S}_{2} \boldsymbol{w}_{+}^{1}\right)(L, y, z)=0, \quad(y, z) \in \mathbb{R}^{2},
$$

and with the Silver-Müller condition on their unbounded part, where $\boldsymbol{w}_{-}^{1}$ and $\boldsymbol{w}_{+}^{2}$ are defined in (2.5) and the operators $\mathcal{S}_{l}, l=1,2$, are general, pseudodifferential operators acting in the $y$ and $z$ directions.

THEOREM 3.2. If the operators $\mathcal{S}_{l}, l=1,2$, have the Fourier symbol

$$
\mathcal{F}\left(\mathcal{S}_{l}\right)=\frac{1}{(\lambda+i \tilde{\omega})(\lambda+i \tilde{\omega}+\sigma Z)}\left[\begin{array}{cc}
k_{y}^{2}-k_{z}^{2}-\lambda \sigma Z & -2 k_{y} k_{z} \\
-2 k_{y} k_{z} & k_{z}^{2}-k_{y}^{2}-\lambda \sigma Z
\end{array}\right],
$$

where $\lambda=\sqrt{|\boldsymbol{k}|^{2}-\tilde{\omega}^{2}+i \tilde{\omega} \sigma Z}$, then the solution of Maxwell's equations (3.1) in $\Omega_{1,2}$ with boundary conditions (3.16) coincides with the restriction on $\Omega_{1,2}$ of the solution of Maxwell's equations (3.1) on $\mathbb{R}^{3}$.

Proof. We show that the difference $\boldsymbol{e}^{i}, i=1,2$, between the solution of the global problem and the solution of the restricted problem vanishes. We consider the case of the second domain; similar computations can be carried out for the first one. The difference $\boldsymbol{e}^{2}$ satisfies in $\Omega_{2}$ the homogeneous counterpart of (3.1) with homogeneous boundary conditions (3.16), and we obtain after a Fourier transform in $y$ and $z$

$$
\hat{\boldsymbol{e}}^{2}=\left(\alpha_{1} \boldsymbol{v}_{1}+\alpha_{2} \boldsymbol{v}_{2}\right) e^{\lambda x}+\left(\alpha_{3} \boldsymbol{v}_{3}+\alpha_{4} \boldsymbol{v}_{4}\right) e^{-\lambda x},
$$

where the vectors $\boldsymbol{v}_{j}, j=1, \ldots, 4$, are defined in (3.8) and (3.9). The Silver-Müller radiation condition implies that $\alpha_{1}=\alpha_{2}=0$. Using now the boundary condition (3.16) at $(0, y, z)$, we obtain that the coefficients $\alpha_{j}, j=3,4$, satisfy the system of equations

$$
\left(A_{1}+\mathcal{S}_{1} A_{2}\right)\left[\begin{array}{l}
\alpha_{3} \\
\alpha_{4}
\end{array}\right]=0
$$

where $A_{1}$ and $A_{2}$ are defined in (3.11). A direct computation with (3.17) leads to

$$
\left[\begin{array}{cc}
-k_{y} k_{z} & k_{y}^{2}-\tilde{\omega}^{2}+i \tilde{\omega} \lambda \\
k_{z}^{2}-\tilde{\omega}^{2}+i \tilde{\omega} \lambda & -k_{y} k_{z}
\end{array}\right]\left[\begin{array}{l}
\alpha_{3} \\
\alpha_{4}
\end{array}\right]=\left[\begin{array}{l}
0 \\
0
\end{array}\right]
$$

which implies $\alpha_{3}=\alpha_{4}=0$. Thus, $\hat{\boldsymbol{e}}^{2}=\mathbf{0}$, which concludes the proof.

Remark 1. As in the case of the Cauchy-Riemann equations, see [13], the symbols in (3.17) can be written in several, mathematically equivalent forms:

$$
\begin{aligned}
\mathcal{F}\left(\mathcal{S}_{l}\right) & =\frac{1}{(\lambda+i \tilde{\omega})(\lambda+i \tilde{\omega}+\sigma Z)} M=\frac{1}{|\boldsymbol{k}|^{2}+\lambda \sigma Z} \frac{\lambda-i \tilde{\omega}}{\lambda+i \tilde{\omega}} M \\
& =\frac{1}{|\boldsymbol{k}|^{2}-\lambda \sigma Z} \frac{\lambda-i \tilde{\omega}-\sigma Z}{\lambda+i \tilde{\omega}+\sigma Z} M=(\lambda-i \tilde{\omega})(\lambda-i \tilde{\omega}-\sigma Z) \tilde{M}^{-1}
\end{aligned}
$$

Copyright $@$ ㅇ by SIAM. Unauthorized reproduction of this article is prohibited. 
where the matrices $M$ and $\tilde{M}$ are given by

$$
M=\left[\begin{array}{cc}
k_{y}^{2}-k_{z}^{2}-\lambda \sigma Z & -2 k_{y} k_{z} \\
-2 k_{y} k_{z} & k_{z}^{2}-k_{y}^{2}-\lambda \sigma Z
\end{array}\right], \quad \tilde{M}=\left[\begin{array}{cc}
k_{y}^{2}-k_{z}^{2}+\lambda \sigma Z & -2 k_{y} k_{z} \\
-2 k_{y} k_{z} & k_{z}^{2}-k_{y}^{2}+\lambda \sigma Z
\end{array}\right] .
$$

This motivates different approximations of the transparent conditions in the context of optimized Schwarz methods. In the case $\sigma=0$, the first form contains a local and a nonlocal term, since multiplication with the matrix $M$ corresponds to second order derivatives in $y$ and $z$, which are local operations, whereas the term containing the square root of $|\boldsymbol{k}|^{2}$ represents a nonlocal operation. The last form contains two nonlocal operations, since the inversion of the matrix $M$ corresponds to an integration. This integration can, however, be passed to the other side of the transmission conditions by multiplication with the matrix $M$. The second form contains two nonlocal terms and a local one. We propose in the next section several approximations based on these different forms and analyze the performance of the associated optimized Schwarz algorithms.

3.4. Optimized Schwarz algorithms for Maxwell's equations. The transparent operators $\mathcal{S}_{l}, l=1,2$, introduced in subsection 3.3, are important in the development of optimized Schwarz methods. When used in algorithm (3.3), they lead to the best possible performance of the method as we will show in Remark 2. The transparent operators are, however, nonlocal operators and, hence, difficult to use in practice. In optimized Schwarz methods, they are, therefore, approximated to obtain practical methods. If one is willing to use second order transmission conditions, then the only parts of the symbols in (3.17) that need to be approximated are the terms $\lambda=\sqrt{|\boldsymbol{k}|^{2}-\tilde{\omega}^{2}+i \tilde{\omega} \sigma Z}$, because the entries of the matrices are polynomials in the Fourier variables, which correspond to derivatives in the $y$ and $z$ directions.

THEOREM 3.3. For the optimized Schwarz algorithm (3.3) with the two subdomain decomposition (3.5), we obtain for $\sigma=0$ the following results:

1. If the operators $\mathcal{S}_{1}$ and $\mathcal{S}_{2}$ have the Fourier symbol

$$
\sigma_{l}:=\mathcal{F}\left(\mathcal{S}_{l}\right)=\gamma_{l}\left[\begin{array}{cc}
k_{y}^{2}-k_{z}^{2} & -2 k_{y} k_{z} \\
-2 k_{y} k_{z} & k_{z}^{2}-k_{y}^{2}
\end{array}\right], \quad \gamma_{l} \in \mathbb{C}\left(k_{z}, k_{y}\right), l=1,2,
$$

then the convergence factor is

$$
\rho=\left|\frac{\left(\sqrt{|k|^{2}-\tilde{\omega}^{2}}-i \tilde{\omega}\right)^{2}}{\left(\sqrt{|k|^{2}-\tilde{\omega}^{2}}+i \tilde{\omega}\right)^{2}} \frac{1-\gamma_{1}\left(\sqrt{|k|^{2}-\tilde{\omega}^{2}}+i \tilde{\omega}\right)^{2}}{1-\gamma_{1}\left(\sqrt{|k|^{2}-\tilde{\omega}^{2}}-i \tilde{\omega}\right)^{2}} \frac{1-\gamma_{2}\left(\sqrt{|k|^{2}-\tilde{\omega}^{2}}+i \tilde{\omega}\right)^{2}}{1-\gamma_{2}\left(\sqrt{|k|^{2}-\tilde{\omega}^{2}}-i \tilde{\omega}\right)^{2}} e^{-2 \sqrt{|k|^{2}-\tilde{\omega}^{2}}} L\right|^{\frac{1}{2}} .
$$

2. If the operators $\mathcal{S}_{1}$ and $\mathcal{S}_{2}$ have the Fourier symbol

$$
\sigma_{l}:=\mathcal{F}\left(\mathcal{S}_{l}\right)=\delta_{l}\left[\begin{array}{cc}
k_{y}^{2}-k_{z}^{2} & -2 k_{y} k_{z} \\
-2 k_{y} k_{z} & k_{z}^{2}-k_{y}^{2}
\end{array}\right]^{-1}, \quad \gamma_{l} \in \mathbb{C}\left(k_{z}, k_{y}\right), \quad l=1,2,
$$

then the convergence factor is

$$
\rho=\left|\frac{\left(\sqrt{|k|^{2}-\tilde{\omega}^{2}}+i \tilde{\omega}\right)^{2}}{\left(\sqrt{|k|^{2}-\tilde{\omega}^{2}}-i \tilde{\omega}\right)^{2}} \frac{\delta_{1}-\left(\sqrt{|\boldsymbol{k}|^{2}-\tilde{\omega}^{2}}-i \tilde{\omega}\right)^{2}}{\delta_{1}-\left(\sqrt{|\boldsymbol{k}|^{2}-\tilde{\omega}^{2}}+i \tilde{\omega}\right)^{2}} \frac{\delta_{2}-\left(\sqrt{|\boldsymbol{k}|^{2}-\tilde{\omega}^{2}}-i \tilde{\omega}\right)^{2}}{\delta_{2}-\left(\sqrt{|\boldsymbol{k}|^{2}-\tilde{\omega}^{2}}+i \tilde{\omega}\right)^{2}} e^{-2 \sqrt{|\boldsymbol{k}|^{2}-\tilde{\omega}^{2}}} L\right|^{\frac{1}{2}} .
$$

Copyright $@$ by SIAM. Unauthorized reproduction of this article is prohibited. 
3. If the operator $\mathcal{S}_{1}$ has the Fourier symbol (3.18) and $\mathcal{S}_{2}$ has the Fourier symbol (3.20), then the convergence factor is

$$
\rho=\left|\frac{1-\gamma_{1}\left(\sqrt{|\boldsymbol{k}|^{2}-\tilde{\omega}^{2}}+i \tilde{\omega}\right)^{2}}{1-\gamma_{1}\left(\sqrt{|\boldsymbol{k}|^{2}-\tilde{\omega}^{2}}-i \tilde{\omega}\right)^{2}} \frac{\delta_{2}-\left(\sqrt{|\boldsymbol{k}|^{2}-\tilde{\omega}^{2}}-i \tilde{\omega}\right)^{2}}{\delta_{2}-\left(\sqrt{|\boldsymbol{k}|^{2}-\tilde{\omega}^{2}}+i \tilde{\omega}\right)^{2}} e^{-2 \sqrt{|\boldsymbol{k}|^{2}-\tilde{\omega}^{2}} L}\right|^{1 / 2} .
$$

Proof. The convergence results are again based on Fourier analysis as in section 3.2. At the $n$th step of the Schwarz algorithm, the coefficients $\boldsymbol{\alpha}=\left(\alpha_{1}, \alpha_{2}\right)$ and $\boldsymbol{\beta}=\left(\beta_{1}, \beta_{2}\right)$ in $(3.10)$ satisfy

$$
\boldsymbol{\alpha}^{n}=\bar{A}_{1}^{-1} \bar{A}_{2} e^{-\lambda L} \boldsymbol{\beta}^{n-1}, \quad \boldsymbol{\beta}^{n}=\bar{B}_{1}^{-1} \bar{B}_{2} e^{-\lambda L} \boldsymbol{\alpha}^{n-1},
$$

where $\lambda=\sqrt{|\boldsymbol{k}|^{2}-\tilde{\omega}^{2}}$ and the matrices $\bar{A}_{l}$ and $\bar{B}_{l}, l=1,2$, are given by

$$
\bar{A}_{1}=A_{1}+\sigma_{1} A_{2}, \quad \bar{A}_{2}=A_{2}+\sigma_{1} A_{1}, \quad \bar{B}_{1}=A_{1}+\sigma_{2} A_{2}, \quad \bar{B}_{2}=A_{2}+\sigma_{2} A_{1},
$$

with $A_{l}, l=1,2$, defined in (3.11). A complete double iteration of the Schwarz algorithm leads, therefore, to

$$
\boldsymbol{\alpha}^{n+1}=\bar{A}_{1}^{-1} \bar{A}_{2} \bar{B}_{1}^{-1} \bar{B}_{2} e^{-2 \lambda L} \boldsymbol{\alpha}^{n-1}, \quad \boldsymbol{\beta}^{n+1}=\bar{B}_{1}^{-1} \bar{B}_{2} \bar{A}_{1}^{-1} \bar{A}_{2} e^{-2 \lambda L} \boldsymbol{\beta}^{n-1} .
$$

Notice that the matrices $A_{1}$ and $A_{2}$ verify the relations

$$
M A_{1}=-(\lambda+i \tilde{\omega})^{2} A_{2}, \quad M A_{2}=-(\lambda-i \tilde{\omega})^{2} A_{1},
$$

which allow us to obtain the convergence factor in all three cases:

1. We obtain, using (3.24),

$$
\begin{aligned}
& \bar{A}_{1}=A_{1}+\gamma_{1} M A_{2}=\left(1-(\lambda-i \tilde{\omega})^{2} \gamma_{1}\right) A_{1}, \\
& \bar{A}_{2}=A_{2}+\gamma_{1} M A_{1}=\left(1-(\lambda+i \tilde{\omega})^{2} \gamma_{1}\right) A_{2}, \\
& \bar{B}_{1}=A_{1}+\gamma_{2} M A_{2}=\left(1-(\lambda-i \tilde{\omega})^{2} \gamma_{2}\right) A_{1}, \\
& \bar{B}_{2}=A_{2}+\gamma_{2} M A_{1}=\left(1-(\lambda+i \tilde{\omega})^{2} \gamma_{2}\right) A_{2},
\end{aligned}
$$

and, therefore, the iteration matrix becomes

$$
R_{1}=\bar{A}_{1}^{-1} \bar{A}_{2} \bar{B}_{1}^{-1} \bar{B}_{2}=\frac{\left(1-\gamma_{1}(\lambda+i \tilde{\omega})^{2}\right)\left(1-\gamma_{2}(\lambda+i \tilde{\omega})^{2}\right)}{\left(1-\gamma_{1}(\lambda-i \tilde{\omega})^{2}\right)\left(1-\gamma_{2}(\lambda-i \tilde{\omega})^{2}\right)}\left(A_{1}^{-1} A_{2}\right)^{2} e^{-2 \lambda L},
$$

and by using the spectral radius of (3.12) the result follows.

2. We get

$$
\begin{aligned}
& \bar{A}_{1}=A_{1}+\delta_{1} M^{-1} A_{2}=\left(1-\frac{\delta_{1}}{(\lambda+i \tilde{\omega})^{2}}\right) A_{1}, \\
& \bar{A}_{2}=A_{2}+\delta_{1} M^{-1} A_{1}=\left(1-\frac{\delta_{1}}{(\lambda-i \tilde{\omega})^{2}}\right) A_{2}, \\
& \bar{B}_{1}=A_{1}+\delta_{2} M^{-1} A_{2}=\left(1-\frac{\delta_{2}}{(\lambda+i \tilde{\omega})^{2}}\right) A_{1}, \\
& \bar{B}_{2}=A_{2}+\delta_{2} M^{-1} A_{1}=\left(1-\frac{\delta_{2}}{(\lambda-i \tilde{\omega})^{2}}\right) A_{2},
\end{aligned}
$$

and, thus, the iteration matrix is

$$
R_{2}=\bar{A}_{1}^{-1} \bar{A}_{2} \bar{B}_{1}^{-1} \bar{B}_{2}=\left(\frac{\lambda+i \tilde{\omega}}{\lambda-i \tilde{\omega}}\right)^{4} \frac{\left(\delta_{1}-(\lambda-i \tilde{\omega})^{2}\right)\left(\delta_{2}-(\lambda-i \tilde{\omega})^{2}\right)}{\left(\delta_{1}-(\lambda+i \tilde{\omega})^{2}\right)\left(\delta_{2}-(\lambda+i \tilde{\omega})^{2}\right)}\left(A_{1}^{-1} A_{2}\right)^{2} e^{-2 \lambda L}
$$

and we use again the spectral radius of (3.12) to conclude.

3 . The conclusion follows as in the first two cases.

Remark 2. From (3.19), we see that the choice $\gamma_{1}=\gamma_{2}=1 /\left(\sqrt{|\boldsymbol{k}|^{2}-\tilde{\omega}^{2}}+i \tilde{\omega}\right)^{2}$ is optimal, since then $\rho \equiv 0$ for all frequencies $\boldsymbol{k}$. With this choice of $\gamma_{1}$ and $\gamma_{2}$, the matrices $\bar{A}_{2}$ and $\bar{B}_{2}$ actually vanish.

Copyright $@$ by SIAM. Unauthorized reproduction of this article is prohibited. 
3.5. A hierarchy of optimized transmission conditions. We present now several particular choices of the remaining parameters in the transmission operators $\mathcal{S}_{l}$ in Theorem 3.3 for $\sigma=0$. To facilitate the use of our results in domain-decomposition codes, we return to the initial notation using the physical parameters $\omega, \varepsilon$, and $\mu$.

Case 1. Taking $\gamma_{1}=\gamma_{2}=0$ in (3.18), which amounts to enforcing the classical characteristic Dirichlet transmission conditions, the convergence factor is

$$
\rho_{1}(\omega, \varepsilon, \mu, L,|\boldsymbol{k}|)=\left|\left(\frac{\sqrt{|\boldsymbol{k}|^{2}-\omega^{2} \varepsilon \mu}-i \omega \sqrt{\varepsilon \mu}}{\sqrt{|\boldsymbol{k}|^{2}-\omega^{2} \varepsilon \mu}+i \omega \sqrt{\varepsilon \mu}}\right)^{2} e^{-2 \sqrt{|\boldsymbol{k}|^{2}-\omega^{2} \varepsilon \mu} L}\right|^{\frac{1}{2}} .
$$

In the nonoverlapping case, $L=0$, this choice ensures convergence only for propagative modes and corresponds to the Taylor transmission conditions of order zero proposed in the seminal paper [11] for the Helmholtz equation.

Case 2. Taking $\gamma_{1}=\gamma_{2}=\frac{1}{|\boldsymbol{k}|^{2}} \frac{s-i \omega \sqrt{\varepsilon \mu}}{s+i \omega \sqrt{\varepsilon \mu}}$ in (3.18) or $\gamma_{1}=\frac{1}{|\boldsymbol{k}|^{2}-2 \omega^{2} \varepsilon \mu+2 i \omega \sqrt{\varepsilon \mu} s}$ in (3.18) and $\delta_{2}=|\boldsymbol{k}|^{2}-2 \omega^{2} \varepsilon \mu-2 i \omega \sqrt{\varepsilon \mu} s$ in (3.20) with $s \in \mathbb{C}$, the convergence factor is

$$
\rho_{2}(\omega, \varepsilon, \mu, L,|\boldsymbol{k}|, s)=\left|\left(\frac{\sqrt{|\boldsymbol{k}|^{2}-\omega^{2} \varepsilon \mu}-s}{\sqrt{|\boldsymbol{k}|^{2}-\omega^{2} \varepsilon \mu}+s}\right)^{2} e^{-2 \sqrt{|\boldsymbol{k}|^{2}-\omega^{2} \varepsilon \mu} L}\right|^{\frac{1}{2}},
$$

which is for $L=0$ identical to the convergence factor obtained for optimized nonoverlapping Schwarz methods for the Helmholtz equation in [24].

Case 3. Taking $\gamma_{1}=\gamma_{2}=\frac{1}{|\boldsymbol{k}|^{2}-2 \omega^{2} \varepsilon \mu+2 i \omega \sqrt{\varepsilon \mu} s}$ in (3.18) with $s \in \mathbb{C}$, the convergence factor is

$$
\begin{aligned}
\rho_{3}(\omega, \varepsilon, \mu, L,|\boldsymbol{k}|, s) & =\left|\frac{\sqrt{|\boldsymbol{k}|^{2}-\omega^{2} \varepsilon \mu}-i \omega \sqrt{\varepsilon \mu}}{\sqrt{|\boldsymbol{k}|^{2}-\omega^{2} \varepsilon \mu}+i \omega \sqrt{\varepsilon \mu}}\right| \rho_{2}(\omega, \varepsilon, \mu, L,|\boldsymbol{k}|, s) \\
& \leq \rho_{2}(\omega, \varepsilon, \mu, L,|\boldsymbol{k}|, s) .
\end{aligned}
$$

Case 4. Taking $\gamma_{l}=\frac{1}{|\boldsymbol{k}|^{2}} \frac{s_{l}-i \omega \sqrt{\varepsilon \mu}}{s_{l}+i \omega \sqrt{\varepsilon \mu}}, l=1,2$, in (3.18) or $\gamma_{1}=\frac{1}{|\boldsymbol{k}|^{2}-2 \omega^{2} \varepsilon \mu+2 i \omega \sqrt{\varepsilon \mu} s_{1}}$ in (3.18) and $\delta_{2}=|\boldsymbol{k}|^{2}-2 \omega^{2} \varepsilon \mu-2 i \omega \sqrt{\varepsilon \mu} s_{2}$ in (3.20) with $s_{l} \in \mathbb{C}, l=1,2$, the convergence factor is

$$
\rho_{4}\left(\omega, \varepsilon, \mu, L,|\boldsymbol{k}|, s_{1}, s_{2}\right)=\left|\frac{\sqrt{|\boldsymbol{k}|^{2}-\omega^{2} \varepsilon \mu}-s_{1}}{\sqrt{|\boldsymbol{k}|^{2}-\omega^{2} \varepsilon \mu}+s_{1}} \frac{\sqrt{|\boldsymbol{k}|^{2}-\omega^{2} \varepsilon \mu}-s_{2}}{\sqrt{|\boldsymbol{k}|^{2}-\omega^{2} \varepsilon \mu}+s_{2}} e^{-2 \sqrt{|\boldsymbol{k}|^{2}-\omega^{2} \varepsilon \mu}}\right|^{\frac{1}{2}},
$$

which is for $L=0$ identical to the convergence factor obtained for a twosided nonoverlapping optimized Schwarz method for the Helmholtz equation in $[22]$.

Case 5. Taking $\gamma_{l}=\frac{1}{|k|^{2}-2 \omega^{2} \varepsilon \mu+2 i \omega \sqrt{\varepsilon \mu} s_{l}}$ in (3.18) with $s_{l} \in \mathbb{C}, l=1,2$, the convergence factor is

$$
\begin{aligned}
\rho_{5}\left(\omega, \varepsilon, \mu, L,|\boldsymbol{k}|, s_{1}, s_{2}\right) & =\left|\frac{\sqrt{|\boldsymbol{k}|^{2}-\omega^{2} \varepsilon \mu}-i \omega \sqrt{\varepsilon \mu}}{\sqrt{|\boldsymbol{k}|^{2}-\omega^{2} \varepsilon \mu}+i \omega \sqrt{\varepsilon \mu}}\right| \rho_{4}\left(\omega, \varepsilon, \mu, L,|\boldsymbol{k}|, s_{1}, s_{2}\right) \\
& \leq \rho_{4}\left(\omega, \varepsilon, \mu, L,|\boldsymbol{k}|, s_{1}, s_{2}\right) .
\end{aligned}
$$

Except for Case 1, all cases use second order transmission conditions, even though we use only a zeroth order approximation of the nonlocal operator $\sqrt{|\boldsymbol{k}|^{2}-\omega^{2} \varepsilon \mu}$. In the cases with parameters, the best choice for the parameters is in general the one that minimizes the convergence factor for all $|\boldsymbol{k}| \in K$, where $K$ denotes the set of relevant 
numerical frequencies. One, therefore, needs to solve the min-max problems

$\min _{s \in \mathbb{C}|\boldsymbol{k}| \in K} \max _{j}(\omega, \varepsilon, \mu, L,|\boldsymbol{k}|, s), j=2,3, \min _{s_{1}, s_{2} \in C|\boldsymbol{k}| \in K} \max _{j} \rho_{j}\left(\omega, \varepsilon, \mu, L,|\boldsymbol{k}|, s_{1}, s_{2}\right), j=4,5$.

We can choose $K=\left[\left(k_{\min }, k_{-}\right) \cup\left(k_{+}, k_{\max }\right)\right]^{2}$, where $k_{\text {min }}$ denotes the smallest frequency relevant to the subdomain, $k_{\max }=\frac{C}{h}$ denotes the largest frequency supported by the numerical grid with mesh size $h$, and $k_{ \pm}$are parameters to be chosen to exclude the resonance frequencies. If, for example, the domain $\Omega$ is a rectilinear conductor with homogeneous Dirichlet conditions on the lateral surface, the solution is the sum of the transverse electric (TE) and transverse magnetic (TM) fields. If the transverse section of the conductor is a rectangle with sides of length $a$ and $b$, the TE and TM fields can be expanded in a Fourier series with the harmonics $\sin \left(\frac{n \pi y}{a}\right) \sin \left(\frac{m \pi z}{b}\right)$, where the relevant frequencies are $|\boldsymbol{k}|=\pi \sqrt{\frac{m^{2}}{a^{2}}+\frac{n^{2}}{b^{2}}}, m, n \in \mathbb{N}^{+}$. The lowest one is, therefore, $k_{\min }=\pi \sqrt{\frac{1}{a^{2}}+\frac{1}{b^{2}}}$, and if the mesh size $h$ satisfies $h=\frac{a}{N}=\frac{b}{M}$, where $N$ and $M$ are the number of grid points in the $y$ and $z$ directions, then the highest frequency would be $k_{\max }=\frac{\sqrt{2} \pi}{h}$. The parameters $k_{ \pm}$would correspond to the frequencies closest to $\omega \sqrt{\varepsilon \mu}$, i.e., $k_{-}=\pi \sqrt{\frac{m_{1}^{2}}{a^{2}}+\frac{n_{1}^{2}}{b^{2}}}$ and $k_{+}=\pi \sqrt{\frac{m_{2}^{2}}{a^{2}}+\frac{n_{2}^{2}}{b^{2}}}$, where $\pi \sqrt{\frac{m_{1}^{2}}{a^{2}}+\frac{n_{1}^{2}}{b^{2}}}<\omega \sqrt{\varepsilon \mu}<\pi \sqrt{\frac{m_{2}^{2}}{a^{2}}+\frac{n_{2}^{2}}{b^{2}}}$, but such precise estimates are not necessary if Krylov acceleration is used; see [24, 22].

The complete mathematical analysis of the min-max problems (3.25) is hard and currently open for $L>0$. When $L=0$, i.e., no overlap, Cases 2 and 4 are equivalent to the corresponding optimized Schwarz method for the Helmholtz equation, for which theoretical results are available; see [22]. Here, we use asymptotic analysis and an equioscillation principle to solve all the min-max problems in (3.25) asymptotically as the mesh size goes to zero, in order to obtain compact formulas for the best parameters to be used in our numerical simulations. This leads to the asymptotic formulas for the optimized parameters of the form $s=p(1-i)$ and $s_{l}=p_{l}(1-i), l=1,2$, with $p$ and $p_{l}$ shown in Table 3.1. These results allow us to compare the performance of

TABLE 3.1

Asymptotic convergence factor and optimal choice of the parameters in the transmission conditions for the five variants of the optimized Schwarz method applied to Maxwell's equations when the mesh parameter $h$ is small, and the maximum numerical frequency is estimated by $k_{\max }=\frac{C}{h}$. Here, $\tilde{\omega}=\omega \sqrt{\varepsilon \mu}$ and $C_{\tilde{\omega}}=\min \left(k_{+}^{2}-\tilde{\omega}^{2}, \tilde{\omega}^{2}-k_{-}^{2}\right)$.

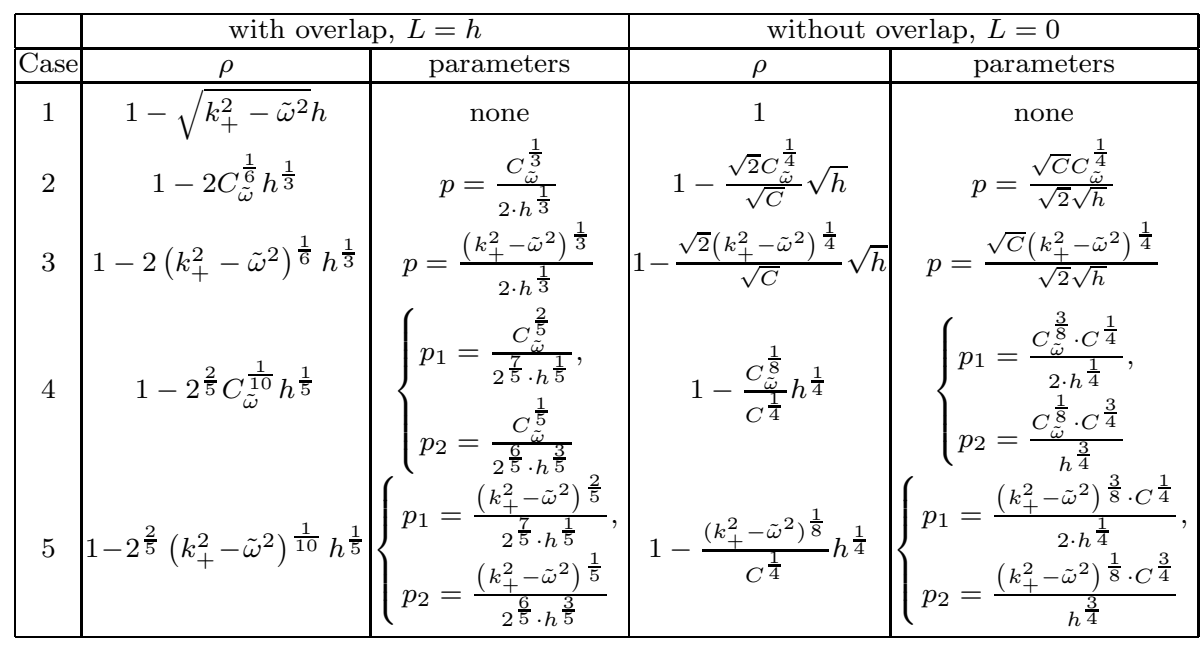

Copyright (c) by SIAM. Unauthorized reproduction of this article is prohibited. 
all the optimized Schwarz methods for Maxwell's equations theoretically: we obtain a hierarchy of better and better convergence factors starting with Case 1 and ending with Case 5. In addition, the explicit formulas for the optimized parameters can be used in order to easily obtain black-box optimized Schwarz methods for Maxwell's equations, which would not be possible otherwise. In section 5 , we will verify these theoretical results numerically.

4. The case of time discretization. If we do not assume the wave to be periodic in time, the time domain also needs to be discretized. We consider a uniform time grid with time step $\Delta t$ and use an implicit time integration scheme for the time derivative in (2.1) of the form

$$
\left\{\begin{array}{l}
-\varepsilon \frac{\boldsymbol{E}^{n+1}-\boldsymbol{E}^{n}}{\Delta t}+\operatorname{curl}\left(\frac{\boldsymbol{H}^{n+1}+\boldsymbol{H}^{n}}{2}\right)-\sigma\left(\frac{\boldsymbol{E}^{n+1}+\boldsymbol{E}^{n}}{2}\right)=\boldsymbol{J}^{n} \\
\mu \frac{\boldsymbol{H}^{n+1}-\boldsymbol{H}^{n}}{\Delta t}+\operatorname{curl}\left(\frac{\boldsymbol{E}^{n+1}+\boldsymbol{E}^{n}}{2}\right)=\mathbf{0}
\end{array}\right.
$$

With this time discretization, we have to solve at each time step the system

$$
-\sigma \boldsymbol{E}-\varepsilon \sqrt{\eta} \boldsymbol{E}+\operatorname{curl} \boldsymbol{H}=\tilde{\boldsymbol{J}}, \quad \mu \sqrt{\eta} \boldsymbol{H}+\operatorname{curl} \boldsymbol{E}=\boldsymbol{g},
$$

where we have set $(\boldsymbol{E}, \boldsymbol{H}):=\left(\boldsymbol{E}^{n+1}, \boldsymbol{H}^{n+1}\right), \sqrt{\eta}:=\frac{2}{\Delta t}, \widetilde{\boldsymbol{J}}:=\boldsymbol{J}^{n}-\sqrt{\eta} \varepsilon \boldsymbol{E}^{n}+2 \sigma \boldsymbol{E}^{n}-$ curl $\boldsymbol{H}^{n}$, and $\boldsymbol{g}=\sqrt{\eta} \mu \boldsymbol{H}^{n}-\operatorname{curl} \boldsymbol{E}^{n}$.

4.1. Classical and optimized Schwarz algorithm. As in the time harmonic case, we consider the problem (4.1) in a bounded domain $\Omega$, with either Dirichlet conditions on the tangent electric field or impedance conditions, on $\partial \Omega$, in order to obtain a well-posed problem; see [30]. For the two subdomain decomposition in Figure 3.1, the classical Schwarz algorithm would at each time step then perform the iteration

$$
\begin{aligned}
-\sqrt{\eta} \varepsilon \boldsymbol{E}^{1, n}+\operatorname{curl} \boldsymbol{H}^{1, n}-\sigma \boldsymbol{E}^{1, n} & =\widetilde{\boldsymbol{J}}^{1} & & \text { in } \Omega_{1}, \\
\sqrt{\eta} \mu \boldsymbol{H}^{1, n}+\operatorname{curl} \boldsymbol{E}^{1, n} & =\boldsymbol{g}^{1} & & \text { in } \Omega_{1}, \\
\mathcal{B}_{\boldsymbol{n}_{1}}\left(\boldsymbol{E}^{1, n}, \boldsymbol{H}^{1, n}\right) & =\mathcal{B}_{\boldsymbol{n}_{1}}\left(\boldsymbol{E}^{2, n-1}, \boldsymbol{H}^{2, n-1}\right) & & \text { on } \Gamma_{12}, \\
-\sqrt{\eta} \varepsilon \boldsymbol{E}^{2, n}+\operatorname{curl} \boldsymbol{H}^{2, n}-\sigma \boldsymbol{E}^{2, n} & =\widetilde{\boldsymbol{J}}^{2} & & \text { in } \Omega_{2}, \\
\sqrt{\eta} \mu \boldsymbol{H}+\operatorname{curl} \boldsymbol{E} & =\boldsymbol{g}^{2} & & \text { in } \Omega_{2}, \\
\mathcal{B}_{\boldsymbol{n}_{2}}\left(\boldsymbol{E}^{2, n}, \boldsymbol{H}^{2, n}\right) & =\mathcal{B}_{\boldsymbol{n}_{2}}\left(\boldsymbol{E}^{1, n-1}, \boldsymbol{H}^{1, n-1}\right) & & \text { on } \Gamma_{21} .
\end{aligned}
$$

TheOREm 4.1. Let $\Omega=\mathbb{R}^{3}$ be decomposed into $\Omega_{1}:=(-\infty, L) \times \mathbb{R}^{2}$ and $\Omega_{2}:=(0,+\infty) \times \mathbb{R}^{2}, L \geq 0$. Then, for any initial guess $\left(\boldsymbol{E}^{1,0} ; \boldsymbol{H}^{1,0}\right) \in\left(L^{2}\left(\Omega_{1}\right)\right)^{6}$, $\left(\boldsymbol{E}^{2,0} ; \boldsymbol{H}^{2,0}\right) \in\left(L^{2}\left(\Omega_{2}\right)\right)^{6}$, the classical Schwarz algorithm (4.2) with overlap $L \geq 0$, including the nonoverlapping case, is for $\sigma \geq 0$ convergent in $\left(L^{2}\left(\Omega_{1}\right)\right)^{6} \times\left(L^{2}\left(\Omega_{2}\right)\right)^{6}$, and the convergence factor is bounded by

$$
R_{c l a}=\frac{\sqrt{L \tilde{\eta}+2 \sqrt{\varepsilon \mu}}-\sqrt{L \tilde{\eta}}}{\sqrt{L \tilde{\eta}+2 \sqrt{\varepsilon \mu}}+\sqrt{L \tilde{\eta}}} e^{-\sqrt{L \eta} \sqrt{L \tilde{\eta}+2 \sqrt{\varepsilon \mu}}}<1,
$$

where $\tilde{\eta}=\eta \varepsilon \mu$.

Proof. This result follows like in the time harmonic case, simply replacing $i \omega$ by $\sqrt{\eta}$. The convergence factor after a Fourier transform is

$$
\rho_{c l a}(|\boldsymbol{k}|)=\left|\frac{\sqrt{|\boldsymbol{k}|^{2}+\eta \varepsilon \mu+\sqrt{\eta} \sigma Z}-\eta \sqrt{\varepsilon \mu}}{\sqrt{|\boldsymbol{k}|^{2}+\eta \varepsilon \mu+\sqrt{\eta} \sigma Z}+\eta \sqrt{\varepsilon \mu}} e^{-\sqrt{|\boldsymbol{k}|^{2}+\eta \varepsilon \mu+\sqrt{\eta} \sigma Z} L}\right|,
$$

Copyright (c) by SIAM. Unauthorized reproduction of this article is prohibited. 
TABLE 4.1

Asymptotic convergence factor and optimal choice of the parameters in the transmission conditions for the five variants of the optimized Schwarz method applied to the time domain Maxwell's equations, when the mesh parameter $h$ is small, and the maximum numerical frequency is estimated by $k_{\max }=\frac{C}{h}$. Here, $\tilde{\eta}=\eta \varepsilon \mu$.

\begin{tabular}{|c|c|c|c|c|}
\hline & \multicolumn{2}{|c|}{ with overlap, $L=h$} & \multicolumn{2}{|c|}{ without overlap, $L=0$} \\
\hline Case & $\rho$ & parameters & $\rho$ & parameters \\
\hline 1 & $1-2^{\frac{3}{2}} \tilde{\eta}^{\frac{1}{4}} \sqrt{h}$ & none & $1-2 \frac{\sqrt{\eta}}{C} h$ & none \\
2 & $1-2^{\frac{13}{6}} \tilde{\eta}^{\frac{1}{6}} h^{\frac{1}{3}}$ & $p=\frac{2^{-\frac{1}{3}} \tilde{\eta}^{\frac{1}{3}}}{h^{\frac{1}{3}}}$ & $1-\frac{4 \tilde{\eta}^{\frac{1}{4}} \sqrt{h}}{\sqrt{C}}$ & $p=\frac{\sqrt{C} \tilde{\eta}^{\frac{1}{4}}}{\sqrt{h}}$ \\
3 & $1-2^{\frac{7}{4}} \tilde{\eta}^{\frac{1}{8}} h^{\frac{1}{4}}$ & $p=\frac{\sqrt{2} \tilde{\eta}^{\frac{1}{4}}}{\sqrt{h}}$ & $1-\frac{2^{\frac{5}{3}} \tilde{\eta}^{\frac{1}{6}}}{C^{\frac{1}{3}}} h^{\frac{1}{3}}$ & $p=\frac{2^{\frac{2}{3}} C^{\frac{2}{3}} \tilde{\eta}^{\frac{1}{6}}}{h^{\frac{2}{3}}}$ \\
4 & $1-2^{\frac{4}{5}} \tilde{\eta}^{\frac{1}{10}} h^{\frac{1}{5}}$ & $p_{1}=\frac{\tilde{\eta}^{\frac{1}{5}}}{2^{\frac{2}{5}} h^{\frac{3}{5}}}, p_{2}=\frac{\tilde{\eta}^{\frac{2}{5}}}{16^{\frac{1}{5}} h^{\frac{1}{5}}}$ & $1-\frac{\sqrt{2} \tilde{\eta}^{\frac{1}{8}}}{C^{\frac{1}{4}}} h^{\frac{1}{4}}$ & $p_{1}=\frac{\sqrt{2} C^{\frac{3}{4}} \tilde{\eta}^{\frac{1}{8}}}{h^{\frac{3}{4}}}, p_{2}=\frac{C^{\frac{1}{4}} \tilde{\eta}^{\frac{3}{8}}}{\sqrt{2} h^{\frac{1}{4}}}$ \\
5 & $1-2^{\frac{7}{6}} \tilde{\eta}^{\frac{1}{12}} h^{\frac{1}{6}}$ & $p_{1}=\frac{2^{\frac{2}{3}} \tilde{\eta}^{\frac{1}{3}}}{h^{\frac{1}{3}}}, p_{2}=\frac{2^{\frac{1}{3}} \tilde{\eta}^{\frac{1}{6}}}{h^{\frac{2}{3}}}$ & $1-\frac{2 \tilde{\eta}^{\frac{1}{10}}}{C^{\frac{1}{5}}} h^{\frac{1}{5}}$ & $p_{1}=\frac{2 C^{\frac{4}{5}} \tilde{\eta}^{\frac{1}{10}}}{h^{\frac{4}{5}}}, p_{2}=\frac{2 C^{\frac{2}{5}} \tilde{\eta}^{\frac{3}{10}}}{h^{\frac{2}{5}}}$ \\
\hline
\end{tabular}

and the method, thus, converges for all Fourier modes. To conclude the proof, it suffices to take the maximum of the convergence factor over $|\boldsymbol{k}|$.

The preceding theorem shows that the classical Schwarz algorithm with Dirichlet transmission conditions applied to the time discretized Maxwell's equations is convergent for all frequencies $|\boldsymbol{k}|$ and that the overlap is not necessary to ensure convergence. The classical Schwarz algorithm corresponds in the case $\sigma=0$ to a simple optimized Schwarz algorithm for the positive definite Helmholtz equation

$$
(\tilde{\eta}-\Delta) u=f,
$$

and from [20] we know that there are much better transmission conditions for such problems. We, thus, propose at each time step the new algorithm

$$
\begin{aligned}
-\sqrt{\eta} \varepsilon \boldsymbol{E}^{1, n}+\operatorname{curl} \boldsymbol{H}^{1, n}-\sigma \boldsymbol{E}^{1, n} & =\widetilde{\boldsymbol{J}}^{1} & & \text { in } \Omega_{1} \\
\sqrt{\eta} \mu \boldsymbol{H}^{1, n}+\operatorname{curl} \boldsymbol{E}^{1, n} & =\boldsymbol{g}^{1} & & \text { in } \Omega_{1} \\
\left(\mathcal{B}_{\boldsymbol{n}_{1}}+\mathcal{S}_{1} \mathcal{B}_{\boldsymbol{n}_{2}}\right)\left(\boldsymbol{E}^{1, n}, \boldsymbol{H}^{1, n}\right) & =\left(\mathcal{B}_{\boldsymbol{n}_{1}}+\mathcal{S}_{1} \mathcal{B}_{\boldsymbol{n}_{2}}\right)\left(\boldsymbol{E}^{2, n-1}, \boldsymbol{H}^{2, n-1}\right) & & \text { on } \Gamma_{12}, \\
-\sqrt{\eta} \varepsilon \boldsymbol{E}^{2, n}+\operatorname{curl} \boldsymbol{H}^{2, n}-\sigma \boldsymbol{E}^{2, n} & =\widetilde{\boldsymbol{J}}^{2} & & \text { in } \Omega_{2}, \\
\sqrt{\eta} \mu \boldsymbol{H}^{2, n}+\operatorname{curl} \boldsymbol{E}^{2, n} & =\boldsymbol{g}^{2} & & \text { in } \Omega_{2}, \\
\left(\mathcal{B}_{\boldsymbol{n}_{2}}+\mathcal{S}_{2} \mathcal{B}_{\boldsymbol{n}_{1}}\right)\left(\boldsymbol{E}^{2, n}, \boldsymbol{H}^{2, n}\right) & =\left(\mathcal{B}_{\boldsymbol{n}_{2}}+\mathcal{S}_{2} \mathcal{B}_{\boldsymbol{n}_{1}}\right)\left(\boldsymbol{E}^{1, n-1}, \boldsymbol{H}^{1, n-1}\right) & & \text { on } \Gamma_{21} .
\end{aligned}
$$

Now Theorem 3.2, Remark 1, Theorem 3.3, and all the cases in subsection 3.5 hold unchanged for the time discretized case of Maxwell's equations upon replacing $i \omega$ by $\sqrt{\eta}$, so we do not restate these results here. The nature of the associated min-max problems (3.25), however, changes fundamentally, and the optimization parameters are now real, $s=p \in \mathbb{R}$ and $s_{l}=p_{l} \in \mathbb{R}, l=1,2$. For Cases 2 and 4 , a complete analysis is available; see [20]. Using a lengthy asymptotic analysis again, we complete the results for the other cases and show in Table 4.1 the asymptotically optimal parameters to use in the time domain case. Again, we obtain an entire hierarchy of optimized Schwarz methods, with better and better convergence factors from Case 1 up to Case 5. While for the time harmonic equations Cases 2 and 3 and Cases 4 and 5 were asymptotically comparable, here all cases are asymptotically different. It is also interesting to note a relationship between the optimized parameters for the time domain case with the one for the Cauchy-Riemann equations; see [13]: Cases 2 and 
4 are identical, since the corresponding convergence rates in the two cases are the same, while for Cases 1, 3, and 5 there is a small difference in the constants, which is due to the additional low frequency term in the Maxwell case. The difference appears to be systematic; the convergence factor of the Maxwell case is obtained from the convergence factor of the Cauchy-Riemann case by replacing $h$ by $2 h$, while for the optimized parameters one has to multiply by 2 , in addition to the replacement of $h$ by $2 h$.

5. Numerical experiments. We discretize the equations using a finite volume method on a staggered grid, which leads to the Yee scheme in the interior. For the first two test cases, we consider the propagation in vacuum with $\varepsilon=\mu=1$ and $\sigma=0$. We first show the two-dimensional problem of transverse electric waves, since this allows us to compute with finer mesh sizes and, thus, to illustrate our asymptotic results by numerical experiments. We simulate directly the error equations, $f=0$, on a uniform mesh with mesh parameter $h$, and we use a random initial guess to ensure that all the frequency components are present in the iteration. We then show the full three-dimensional (3d) case, first for a model problem, and then for the application of heating a chicken in a microwave oven.

5.1. Two-dimensional case. We consider the TE waves problem in the plane $(x, y, 0)$. There is no more dependence on $z$, and the components $E_{3}, H_{1}$, and $H_{2}$ are identically zero. The problem obtained is formally identical to the three-dimensional case (2.3) if $\boldsymbol{u}=\left(E_{1}, E_{2}, H_{3}\right)^{t}$, and the matrix $N_{\mathbf{v}}$ becomes

$$
N_{\mathbf{v}}=\left(\begin{array}{c}
-v_{y} \\
v_{x}
\end{array}\right)
$$

and the matrices $G_{x}, G_{y}$, and $G_{\mathbf{v}}$ are

$$
G_{x}=\left(\begin{array}{cc} 
& N_{\mathbf{e}_{x}} \\
N_{\mathbf{e}_{x}}^{t} &
\end{array}\right), \quad G_{y}=\left(\begin{array}{cc} 
& N_{\mathbf{e}_{y}} \\
N_{\mathbf{e}_{y}}^{t} &
\end{array}\right), \quad \text { and } \quad G_{\mathbf{v}}=\left(\begin{array}{cc} 
& N_{\mathbf{v}} \\
N_{\mathbf{v}}^{t} &
\end{array}\right) .
$$

All the analytical results remain valid we need only to replace $|\boldsymbol{k}|$ by $\left|k_{y}\right|$ and the corresponding quantities in the optimized parameters for both time harmonic and time discretized solutions. We solve Maxwell's equations on the unit square $\Omega=$ $(0,1)^{2}$ with a zeroth order approximation of the absorbing boundary conditions on $\partial \Omega$. The domain $\Omega$ is decomposed into the two subdomains $\Omega_{1}=(0, \beta) \times(0,1)$ and $\Omega_{2}=(\alpha, 1) \times(0,1)$, where $0<\alpha \leq \beta<1$, and, therefore, the overlap is $L=\beta-\alpha$, and we consider both decompositions with and without overlap.

In the time harmonic case, the frequency $\tilde{\omega}=2 \pi$ is chosen such that the rule of thumb of 10 points per wavelength is not violated. Table 5.1 shows the iteration

TABLE 5.1

Number of iterations in the $2 d$ time harmonic case to attain a relative residual reduction of $10^{-6}$ for different transmission conditions and different mesh sizes.

\begin{tabular}{|c|c|c|c|c|c|c|c|c|}
\hline & \multicolumn{3}{|c|}{ with overlap, $L=h$} & \multicolumn{4}{c|}{ without overlap, $L=0$} \\
\hline Case 1 & $1 / 32$ & $1 / 64$ & $1 / 128$ & $1 / 256$ & $1 / 32$ & $1 / 64$ & $1 / 128$ & $1 / 256$ \\
Case 2 & $19(14)$ & $47(27)$ & $72(33)$ & $118(45)$ & $-(73)$ & $-(100)$ & $-(138)$ & $-(181)$ \\
Case 3 & $13(13)$ & $14(14)$ & $22(17)$ & $26(19)$ & $41(26)$ & $57(34)$ & $79(40)$ & $111(47)$ \\
Case 4 & $19(14)$ & $21(16)$ & $24(18)$ & $21(18)$ & $41(23)$ & $56(25)$ & $80(28)$ & $115(35)$ \\
Case 5 & $13(13)$ & $15(15)$ & $17(18)$ & $19(19)$ & $31(24)$ & $35(28)$ & $41(30)$ & $47(33)$ \\
\hline
\end{tabular}



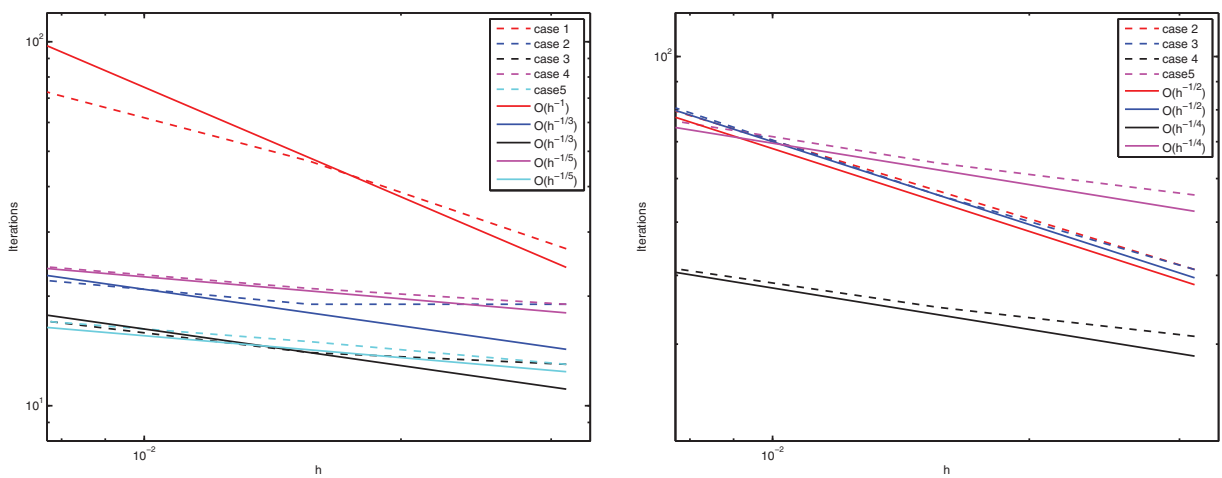

FIG. 5.1. Asymptotics for the overlapping (left) and nonoverlapping (right) cases for the time harmonic equations.

TABLE 5.2

Number of iterations in the $2 d$ time discretized case to attain a relative residual reduction of $10^{-6}$ for different transmission conditions and different mesh sizes.

\begin{tabular}{|c|c|c|c|c|c|c|c|c|}
\hline & \multicolumn{3}{|c|}{ with overlap, $L=h$} & \multicolumn{4}{c|}{ without overlap, $L=0$} \\
\hline$h$ & $1 / 16$ & $1 / 32$ & $1 / 64$ & $1 / 128$ & $1 / 16$ & $1 / 32$ & $1 / 64$ & $1 / 128$ \\
\hline Case 1 & $17(10)$ & $25(12)$ & $35(14)$ & $47(17)$ & $282(21)$ & $574(27)$ & $1122(33)$ & $2244(41)$ \\
Case 2 & $14(8)$ & $16(9)$ & $20(11)$ & $25(12)$ & $40(14)$ & $59(18)$ & $79(21)$ & $111(24)$ \\
Case 3 & $12(8)$ & $14(9)$ & $16(9)$ & $18(9)$ & $14(9)$ & $17(11)$ & $20(12)$ & $25(14)$ \\
Case 4 & $12(9)$ & $14(10)$ & $16(12)$ & $19(13)$ & $21(13)$ & $27(15)$ & $33(17)$ & $39(19)$ \\
Case 5 & $12(8)$ & $14(9)$ & $15(10)$ & $17(10)$ & $14(9)$ & $17(11)$ & $17(12)$ & $19(13)$ \\
\hline
\end{tabular}

count to achieve a relative residual reduction of $10^{-6}$ for all Schwarz algorithms we considered in the overlapping and nonoverlapping cases. The results are presented in the form $i t_{S}\left(i t_{G M}\right)$, where $i t_{S}$ denotes the iteration number for the iterative version of the algorithm and $i t_{G M}$ the iteration number for the accelerated version using GMRES, and a dash means no convergence.

In Figure 5.1 we show the results we obtained in a graph, together with the expected asymptotics. Both on the left in the overlapping case and on the right in the nonoverlapping one, the asymptotics agree quite well, except for the classical case with overlap, where the algorithm performs better than predicted by the asymptotic analysis. In the case of the Cauchy-Riemann equations [13], it was observed that certain discretizations of the hyperbolic system can introduce higher order terms in the discretized transmission conditions, which can improve the convergence behavior, as we observe it here, an issue that merits further study.

For the time discretized Maxwell's equations, we choose $\tilde{\eta}=1$. Table 5.2 shows the iteration count for one time step to achieve a relative residual reduction of $10^{-6}$ for all Schwarz algorithms we considered in the overlapping and nonoverlapping cases. We observe that the classical nonoverlapping algorithm converges only very slowly; the need of optimized methods is evident here.

In Figure 5.2 we show the results we obtained in a graph, together with the expected asymptotics, and there is very good agreement.

5.2. Three-dimensional case. We solve now Maxwell's equations on the unit cube $\Omega=(0,1)^{3}$. We decompose the domain into two subdomains $\Omega_{1}=(0, \beta) \times(0,1)^{2}$ and $\Omega_{2}=(\alpha, 1) \times(0,1)^{2}$, with $0<\alpha \leq \beta<1$, and $L=\beta-\alpha$ as before. In the time harmonic case, we chose the frequency $\tilde{\omega}=2 \pi / 3$ to satisfy the rule of thumb of 

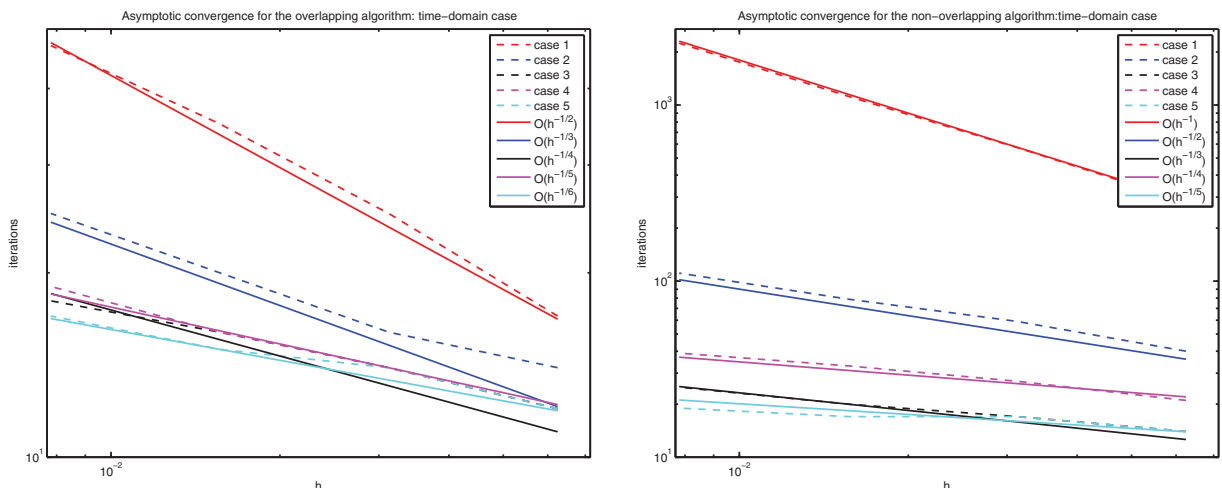

FIG. 5.2. Asymptotics for the overlapping (left) and nonoverlapping (right) cases for the time discretized equations.

TABLE 5.3

Number of iterations in the $3 d$ time harmonic case to attain a relative residual reduction of $10^{-6}$ for different transmission conditions and different mesh sizes.

\begin{tabular}{|c|c|c|c|c|c|c|}
\hline & \multicolumn{3}{|c|}{ with overlap, $L=h$} & \multicolumn{3}{c|}{ without overlap, $L=0$} \\
\hline$h$ & $1 / 8$ & $1 / 16$ & $1 / 30$ & $1 / 8$ & $1 / 16$ & $1 / 30$ \\
\hline Case 1 & $19(13)$ & $29(17)$ & $46(22)$ & $-(93)$ & $-(140)$ & $-(202)$ \\
Case 2 & $14(12)$ & $19(14)$ & $23(16)$ & $48(29)$ & $69(36)$ & $98(48)$ \\
Case 3 & $16(12)$ & $18(14)$ & $21(16)$ & $65(35)$ & $80(42)$ & $166(55)$ \\
Case 4 & $15(13)$ & $19(15)$ & $22(17)$ & $38(28)$ & $60(33)$ & $104(39)$ \\
Case 5 & $16(13)$ & $18(14)$ & $21(16)$ & $70(36)$ & $80(42)$ & $176(55)$ \\
\hline
\end{tabular}

TABLE 5.4

Number of iterations in the $3 d$ time discretized case to attain a relative residual reduction of $10^{-6}$ for different transmission conditions and different mesh sizes.

\begin{tabular}{|c|c|c|c|c|c|c|}
\hline & \multicolumn{3}{|c|}{ with overlap, $L=h$} & \multicolumn{3}{c|}{ without overlap, $L=0$} \\
\hline$h$ & $1 / 8$ & $1 / 16$ & $1 / 30$ & $1 / 8$ & $1 / 16$ & $1 / 30$ \\
\hline Case 1 & $14(8)$ & $18(10)$ & $25(12)$ & $246(32)$ & $467(43)$ & $859(70)$ \\
Case 2 & $13(8)$ & $18(10)$ & $22(11)$ & $46(24)$ & $65(37)$ & $87(65)$ \\
Case 3 & $12(8)$ & $15(9)$ & $17(10)$ & $47(23)$ & $59(28)$ & $73(60)$ \\
Case 4 & $14(9)$ & $17(10)$ & $19(11)$ & $48(21)$ & $57(33)$ & $66(63)$ \\
Case 5 & $12(8)$ & $14(9)$ & $16(10)$ & $46(24)$ & $53(29)$ & $60(60)$ \\
\hline
\end{tabular}

10 points per wavelength. Table 5.3 shows the iteration count to achieve a relative residual reduction of $10^{-6}$ for all Schwarz algorithms we considered, both in the overlapping and nonoverlapping cases.

The results for the time discretized Maxwell's equations where $\tilde{\eta}=1$ are shown in Table 5.4.

5.3. A three-dimensional application: Chicken in a microwave oven. We apply now the previous principles to derive an efficient domain-decomposition method based on optimized interface conditions to solve a realistic application: heating up a chicken in a microwave oven; see Figure 5.3 on the left. The computational domain is now given by the heating cavity of a Whirlpool Talent Combi 4 microwave oven, $\Omega=[0,0.32] \times[0,0.36] \times[0,0.20]$ meters. We impose metallic boundary conditions (which means a null tangential electric field) on all faces except on the right of the 

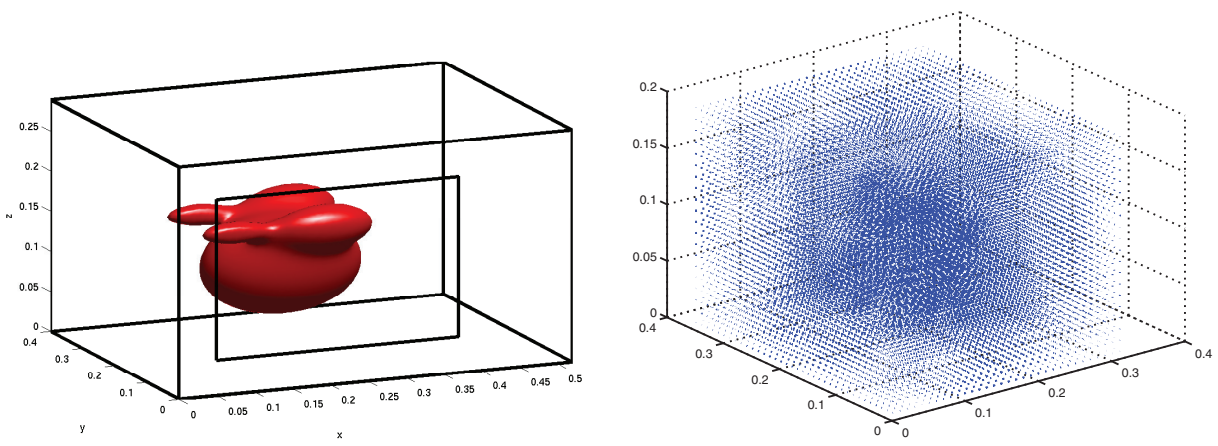

FIG. 5.3. Chicken in a Whirlpool Talent Combi 4 microwave oven on the left and the real part of the magnetic field in the cooking cavity while heating the chicken on the right.
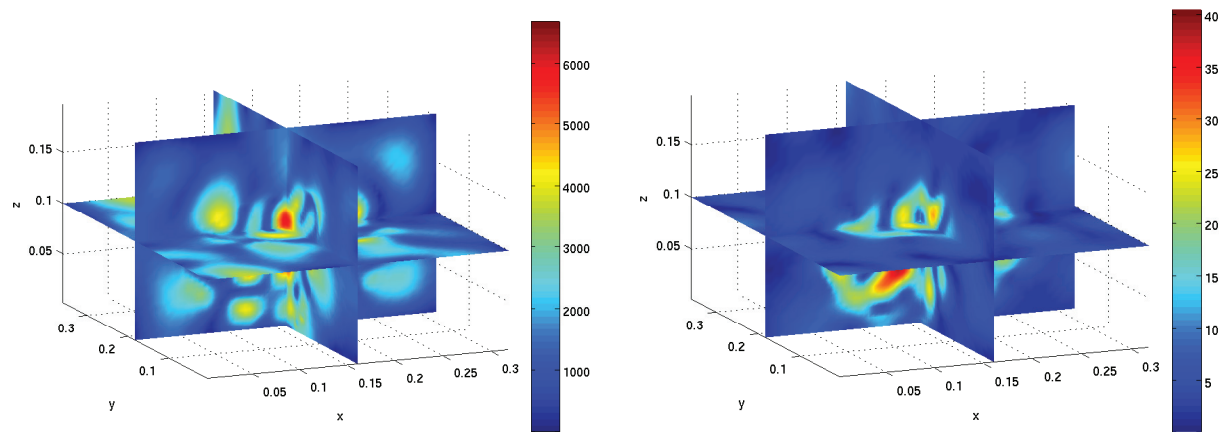

FIG. 5.4. Chicken heating in a microwave oven: electric field intensity on the left and magnetic field intensity on the right.

oven, where the components of the electric field are the dominant TE10 mode generated by the magnetron on a small rectangle of dimensions $0.08 \times 0.04$. The electric and electromagnetic properties of the media are now nonconstant in the computational domain: inside the chicken, we have an electric permittivity $\varepsilon=4.43 \cdot 10^{-11} \frac{\mathrm{farad}}{\mathrm{m}}$ and the conductivity is $\sigma=3 \cdot 10^{-11 \frac{\text { siemens }}{\mathrm{m}}}$, whereas for the air $\varepsilon=8.85 \cdot 10^{-12 \frac{\mathrm{farad}}{\mathrm{m}}}$ and $\sigma=0 \frac{\text { siemens }}{\mathrm{m}}$. The magnetic permeability is the same for both, $\mu=4 \pi \cdot 10^{-7} \frac{\mathrm{m} \text { henry }}{\mathrm{m}}$, and the frequency is given by $\omega=2 \pi \cdot 2.45 \mathrm{GHz}$.

We decompose the microwave oven into $2 \times 2 \times 2=8$ subdomains of equal size on a grid with mesh size $h=0.005$, which allows us to solve this problem on a personal computer, where a direct factorization would not have been possible anymore. This resolution is enough for the wavelength of the microwave and also for most of the geometry of the chicken, except maybe for the drumstick tips. The real part of the magnetic field of the solution is shown in Figure 5.3 on the right, and the intensity (Euclidian norm) of the electric and magnetic fields in the oven are shown in Figure 5.4 in three dimensions. Two-dimensional cross sections of the solution are shown in Figure 5.5 , where we show in each row on the left the electric and on the right the magnetic field intensities. One can see from these computational experiments why a turntable is so important in a microwave oven: there are hot spots, where the intensity of the standing wave is high in the chicken, and other areas, where there is very little heating happening. Only a turntable can lead to an approximately even heating of the chicken. 



FIG. 5.5. Cross sections of the electric and magnetic field intensities.

6. Conclusions. We have shown that, for Maxwell's equations, a classical Schwarz algorithm using characteristic Dirichlet transmission conditions between subdomains has the same convergence behavior as a simple optimized Schwarz method applied to the Helmholtz equation, with a low frequency approximation of the optimal transmission conditions. This relation allowed us to develop easily an entire hierarchy of optimized overlapping and nonoverlapping Schwarz methods with better transmission conditions than the characteristic ones for Maxwell's equations. We illustrated with numerical experiments that the new algorithms converge much more rapidly than the classical one and that such algorithms can be effectively used to compute an approximate solution for a large scale application. This latter problem contains a positive conductivity, variable coefficients, and multiple subdomains, a case which is not covered by our current analysis. Nevertheless, the algorithm performs well with the coefficients derived from the zero conductivity, constant coefficient case. We are 
currently studying the optimization problem with nonzero conductivity, for which the equivalence with the Helmholtz equation does not hold any longer.

The equivalence between systems and scalar equations has already been instrumental for the development of optimized Schwarz algorithms for the Cauchy-Riemann equations and will almost certainly play an important role for other cases. For example, it was observed in [15] that, for Euler's equation, the classical Schwarz algorithm with characteristic information exchange at the interfaces is convergent, even without overlap. To relate systems of partial differential equations to scalar ones, the algebraic tool of the Smith factorization [40] has proved to be useful; see [16].

Acknowledgment. The second author acknowledges the support of the Swiss National Science Foundation grants 200020-117577/1 and 200021-107988.

\section{REFERENCES}

[1] A. Alonso Rodriguez and L. Gerardo-Giorda, New nonoverlapping domain decomposition methods for the harmonic Maxwell system, SIAM J. Sci. Comput., 28 (2006), pp. 102-122.

[2] S. Benzoni-Gavage And D. Serre, Multi-dimensional Hyperbolic Partial Differential Edquations: First-order Systems and Applications, Oxford Math. Monogr., Oxford Science Publications, New York, 2007.

[3] T. F. Chan and T. P. Mathew, Domain decomposition algorithms, in Acta Numerica 1994, Vol. 3, Cambridge University Press, New York, 1994, pp. 61-143.

[4] P. Charton, F. Nataf, And F. Rogier, Méthode de décomposition de domaine pour l'équation d'advection-diffusion, C. R. Acad. Sci. Paris Ser. I, 313 (1991), pp. 623-626.

[5] P. Chevalier, Méthodes Numériques Pour les Tubes Hyperfréquences. Résolution par Décomposition de Domaine, Ph.D. thesis, Université Paris VI, Paris, 1998.

[6] P. Chevalier And F. NATAF, Symmetrized method with optimized second-order conditions for the Helmholtz equation, in Domain Decomposition Methods 10, The Tenth International Conference on Domain Decomposition Methods (Boulder, CO, 1997), Amer. Math. Soc., Providence, RI, 1998, pp. 400-407.

[7] S. Clerc, Non-overlapping Schwarz method for systems of first order equations, Contemp. Math., 218 (1998), pp. 408-416.

[8] P. Collino, G. Delbue, P. Joly, and A. Piacentini, A new interface condition in the nonoverlapping domain decomposition, Comput. Methods Appl. Mech. Engrg., 148 (1997), pp. 195-207.

[9] Q. Deng, Timely communication: An analysis for a nonoverlapping domain decomposition iterative procedure, SIAM J. Sci. Comput., 18 (1997), pp. 1517-1525.

[10] B. Després, Décomposition de domaine et problème de Helmholtz, C. R. Acad. Sci. Paris, 1 (1990), pp. 313-316.

[11] B. Després, Domain decomposition method and the Helmholtz problem. II, in Second International Conference on Mathematical and Numerical Aspects of Wave Propagation (Newark, DE, 1993), SIAM, Philadelphia, PA, 1993, pp. 197-206.

[12] B. Després, P. Joly, And J. E. Roberts, A domain decomposition method for the harmonic Maxwell equations, in Iterative Methods in Linear Algebra: Proceedings (Brussels, 1991), North-Holland, Amsterdam, 1992, pp. 475-484.

[13] V. Dolean and M. J. Gander, Why classical Schwarz methods applied to hyperbolic systems converge even without overlap, in Domain Decomposition Methods in Science and Engineering XVII, Seventeenth International Conference on Domain Decomposition Methods, Lect. Notes Comput. Sci. Eng. 60, Springer, Berlin, 2007, pp. 467-476.

[14] V. Dolean, S. Lanteri, And F. Nataf, Construction of interface conditions for solving compressible Euler equations by non-overlapping domain decomposition methods, Internat. J. Numer. Methods Fluids, 40 (2002), pp. 1485-1492.

[15] V. Dolean, S. Lanteri, And F. NATAF, Convergence analysis of a Schwarz type domain decomposition method for the solution of the Euler equations, Appl. Numer. Math., 49 (2004), pp. 153-186.

[16] V. Dolean, G. Rapin, And F. Nataf, Deriving a new domain decomposition method for the Stokes equations using the Smith factorization, Math. Comp., 78 (2009), pp. 789-814.

[17] B. Engquist And H.-K. ZhaO, Absorbing boundary conditions for domain decomposition, Appl. Numer. Math., 27 (1998), pp. 341-365. 
[18] E. Faccioli, F. Maggio, A. Quarteroni, and A. Tagliani, Spectral domain decomposition methods for the solution of acoustic and elastic wave propagation, Geophys., 61 (1996), pp. 1160-1174.

[19] E. Faccioli, F. Maggio, A. Quarteroni, and A. Tagliani, $2 d$ and $3 d$ elastic wave propagation by pseudo-spectral domain decomposition method, J. Seismology, 1 (1997), pp. 237-251.

[20] M. J. Gander, Optimized Schwarz methods, SIAM J. Numer. Anal., 44 (2006), pp. 699-731.

[21] M. J. Gander and L. Halpern, Méthodes de relaxation d'ondes pour l'équation de la chaleur en dimension 1, C. R. Acad. Sci. Paris Sér. I, 336 (2003), pp. 519-524.

[22] M. J. Gander, L. Halpern, and F. Magoulès, An optimized Schwarz method with two-sided Robin transmission conditions for the Helmholtz equation, Internat. J. Numer. Methods Fluids, 55 (2007), pp. 163-175.

[23] M. J. Gander, L. HAlPERn, And F. NATAF, Optimal Schwarz waveform relaxation for the one dimensional wave equation, SIAM J. Numer. Anal., 41 (2003), pp. 1643-1681.

[24] M. J. Gander, F. Magoulès, And F. NAtaf, Optimized Schwarz methods without overlap for the Helmholtz equation, SIAM J. Sci. Comput., 24 (2002), pp. 38-60.

[25] T. Hagstrom and S. Lau, Radiation boundary conditions for Maxwell's equations: A review of accurate time-domain formulations, J. Comput. Math, 25 (2007), pp. 305-336.

[26] T. Hagstrom, R. P. Tewarson, and A. Jazcilevich, Numerical experiments on a domain decomposition algorithm for nonlinear elliptic boundary value problems, Appl. Math. Lett., 1 (1988), pp. 299-302.

[27] C. Japhet, F. NAtAF, And F. Rogier, The optimized order 2 method. Application to convection-diffusion problems, Future Generation Comput. Systems, 18 (2001), pp. 1730 .

[28] P.-L. Lions, On the Schwarz alternating method. III: A variant for nonoverlapping subdomains, in Third International Symposium on Domain Decomposition Methods for Partial Differential Equations, Houston, TX, 1989, T. F. Chan, R. Glowinski, J. Périaux, and O. Widlund, eds., SIAM, Philadelphia, PA, 1990, pp. 202-223.

[29] F. NATAF And F. Rogier, Factorization of the convection-diffusion operator and the Schwarz algorithm, Math. Models Methods Appl. Sci., 5 (1995), pp. 67-93.

[30] J.-C. Nedelec, Acoustic and Electromagnetic Equations. Integral Representations for Harmonic Problems, Appl. Math. Sci. 144, Springer, New York, 2001.

[31] A. Quarteroni, Domain decomposition methods for systems of conservation laws: Spectral collocation approximations, SIAM J. Sci. Stat. Comput., 11 (1990), pp. 1029-1052.

[32] A. Quarteroni And L. Stolcis, Homogeneous and heterogeneous domain decomposition methods for compressible fluid flows at high Reynolds numbers, CFD Rev., II (1998), pp. 10641078.

[33] A. Quarteroni and A. Valli, Domain Decomposition Methods for Partial Differential Equations, Oxford Science Publications, New York, 1999.

[34] B. F. Smith, P. E. Buørstad, And W. Gropp, Domain Decomposition: Parallel Multilevel Methods for Elliptic Partial Differential Equations, Cambridge University Press, Cambridge, 1996.

[35] I. Sofronov, Nonreflecting inflow and outflow in a wind tunnel for transonic time-accurate simulation, J. Math. Anal. Appl., 221 (1998), pp. 92-115.

[36] H. Sun And W.-P. TANG, An overdetermined Schwarz alternating method, SIAM J. Sci. Comput., 17 (1996), pp. 884-905.

[37] W. P. TANG, Generalized Schwarz splittings, SIAM J. Sci. Stat. Comp., 13 (1992), pp. 573-595.

[38] A. ToselLI, Overlapping Schwarz methods for Maxwell's equations in three dimensions, Numer. Math., 86 (2000), pp. 733-752.

[39] A. Toselli and O. Widlund, Domain Decomposition Methods - Algorithms and Theory, Springer Ser. Comput. Math. 34, Springer, New York, 2004.

[40] J. Wloka, B. Rowley, And B. Lawruk, Boundary Value Problems for Elliptic Systems, Cambridge University Press, New York, 1995.

[41] J. Xu, Iterative methods by space decomposition and subspace correction, SIAM Rev., 34 (1992), pp. 581-613.

[42] J. Xu AND J. Zou, Some nonoverlapping domain decomposition methods, SIAM Rev., 40 (1998), pp. 857-914.

Copyright $@$ by SIAM. Unauthorized reproduction of this article is prohibited. 